\title{
Uma abordagem bayesiana para alguns modelos de crescimento na presença de assimetria e heteroscedasticidade
}


Data de Depósito:

Assinatura:

\title{
Uma abordagem bayesiana para alguns modelos de crescimento na presença de assimetria e heteroscedasticidade
}

\author{
Aline Minniti de Campos
}

Orientador: Prof. Dr. Marinho Gomes de Andrade Filho

Dissertação apresentada ao Instituto de Ciências Matemáticas e de Computação - ICMC-USP, como parte dos requisitos para obtenção do título de Mestre em Ciências - Ciências de Computação e Matemática Computacional. VERSÃO REVISADA

USP - São Carlos

Outubro/2011 
Ficha catalográfica elaborada pela Biblioteca Prof. Achille Bassi e Seção Técnica de Informática, ICMC/USP, com os dados fornecidos pelo(a) autor(a)

Minniti de Campos, Aline
Uma abordagem bayesiana para alguns modelos de
crescimento na presença de assimetria e
heteroscedasticidade / Aline Minniti de Campos;
orientador Marinho Gomes de Andrade Filho -- São
Carlos, 2011.
63 p.
Dissertação (Mestrado - Programa de Pós-Graduação en
Ciências de Computação e Matemática Computacional) --
Instituto de Ciências Matemáticas e de Computação,
Universidade de São Paulo, 2011.
1. Inferência bayesiana. 2. Modelos de
crescimento. 3. Assimetria. 4. Heteroscedasticidade.
I. Gomes de Andrade Filho, Marinho, orient. II.
Título.




\section{Agradecimentos}

Primeiramente agradeço a Deus, que me dá saúde, força e paciência para superar os obstáculos todos os dias.

Aos meus pais, que me apoiam e incentivam em todos os momentos de minha vida e a quem sempre terei imensa gratidão. A minha irmã, pela amizade constante e pelas etapas vividas em São Carlos.

Ao meu orientador, Marinho Gomes de Andrade Filho, por ter acreditado em mim e por estar presente em cada etapa deste trabalho. Agradeço ainda pela paciência e incentivo na elaboração e condução deste trabalho. Minha eterna gratidão e admiração.

Ao professor Vicente Garibay Cancho pelas diretrizes e sugestões, sempre acrescentando sábias informações.

Aos professores Jorge Achcar e Mário de Castro, membros da banca do exame de qualificação, pelas sugestões feitas.

Aos professores e funcionários do ICMC pela minha formação profissional, pessoal e pelo excelente convívio.

A todos os meus amigos do curso de graduação e pós-graduação pelos momentos compartilhados no decorrer destes anos. A ajuda e amizade de vocês foram fundamentais para meu crescimento nesta jornada. Não citarei nomes, pois a lista seria muito extensa para ser colocada aqui.

A todas as pessoas que não foram nominalmente mencionadas, mas que de alguma forma contribuíram para viabilizar este trabalho.

Finalmente, agradeço à Coordenação de Aperfeiçoamento de Pessoal de Nível Superior (CAPES) pelo suporte financeiro concedido para a realização deste projeto. 


\section{Resumo}

Esta dissertação flexibiliza a suposição de normalidade, dispondo de distribuições assimétricas em modelos de crescimento. Propõe uma abordagem bayesiana para ajuste de modelos não lineares quando a suposição de normalidade para os erros não é razoável e/ou apresentam heteroscedasticidade. Assim, adota-se as distribuições skew-normal e skew-t para as situações em que é necessário modelar dados com caudas mais pesadas ou mais leves que a normal e assimétricos; sendo que é considerado também a presença de heteroscedasticidade. Diferentes funções são utilizadas na estrutura multiplicativa para modelar a variância. Com esse objetivo, métodos de inferência na abordagem bayesiana são desenvolvidos para estimar os parâmetros dos modelos de regressão não linear com os erros seguindo as distribuições citadas anteriormente. A metodologia visa aplicação à curvas de crescimento para dados de árvores.

Palavras-chave: Inferência Bayesiana, modelos de crescimento, assimetria, heteroscedasticidade. 


\begin{abstract}
This paper relaxes the assumption of normality, featuring asymmetric distributions in growth models. Proposes a Bayesian approach to fit nonlinear models when the assumption of normality for the errors is not reasonable and/or exhibit heteroscedasticity. Thus, we adopt the skew-normal and skew-t distributions for situations where it is necessary to model data with tails heavier or lighter than normal and asymmetric, which is considered also the presence of heteroscedasticity. Different functions are used to model the multiplicative structure of variance. With this objective, methods of inference in the Bayesian approach are developed to estimate the parameters of nonlinear regression models with errors following the distributions listed above. The methodology is intended to apply to the growth curves for trees data sets.
\end{abstract}

Keywords: Bayesian inference, growth curves, asymmetry, heteroscedasticity. 


\section{Lista de Figuras}

2.1 Curvas de crescimento. . . . . . . . . . . . . . . . . . . . 12

3.1 Funções de densidade para a distribuição skew normal padrão, com diferentes valores para $\lambda \ldots \ldots \ldots \ldots \ldots$

3.2 Funções de densidade para a distribuição skew t padrão, com diferentes valores para $\lambda$ e $\nu \ldots \ldots \ldots \ldots \ldots \ldots$

6.1 Histograma e normal Q-Q plot dos dados arbóreos. . . . . . . . . . . . 39

6.2 Histogramas dos parâmetros do modelo de Brody com erros skew normal. 44

6.3 Série dos parâmetros do modelo de Brody com erros skew normal. . . . 44

6.4 Histogramas dos parâmetros do modelo de Brody heteroscedástico com erros skew normal. . . . . . . . . . . . . . . . . . . . . . . 48

6.5 Série dos parâmetros do modelo de Brody heteroscedástico com erros skew normal. . . . . . . . . . . . . . . . . . . . . . 49

6.6 Modelo ajustado para os dados arbóreos. . . . . . . . . . . . . . . . . 49

6.7 Dados com a pertubação de uma observação. . . . . . . . . . . . . . . 50

6.8 Divergência de Kullback-Leibler. . . . . . . . . . . . . . . . . . . 51

6.9 Calibração da divergência K-L . . . . . . . . . . . . . . . . . . . . . 52 


\section{Lista de Tabelas}

6.1 Sumário dos critérios de seleção com erros normais . . . . . . . . . . 38

6.2 Sumário do modelo para os dados simulados . . . . . . . . . . . . . 38

6.3 Sumário dos critérios de seleção com erros normais . . . . . . . . . . . 41

6.4 Sumário do modelo de Brody com erros normais . . . . . . . . . . . . . 41

6.5 Sumário dos critérios de seleção com erros t-Student . . . . . . . . . . . 41

6.6 Sumário do modelo de Brody com erros t-Student . . . . . . . . . . . . 42

6.7 Sumário dos critérios de seleção com erros skew normal . . . . . . . . . 42

6.8 Sumário do modelo de Brody com erros skew normal . . . . . . . . . . 42

6.9 Sumário dos critérios de seleção com erros skew-t . . . . . . . . . . . . 43

6.10 Sumário do modelo de Brody com erros skew-t . . . . . . . . . . . . . . 43

6.11 Sumário do modelo de Brody com erros skew normal e função de variância $m_{i}=\exp \left(\rho x_{i}\right) \ldots \ldots \ldots \ldots$

6.12 Sumário do modelo de Brody com erros skew normal e função de variância $m_{i}=x_{i}^{\rho} \ldots \ldots \ldots \ldots \ldots$

6.13 Sumário dos critérios de seleção para os modelos heteroscedásticos com erros skew normal . . . . . . . . . . . . . . . . . . . 4 
6.14 Sumário do modelo de Brody com erros skew t e função de variância

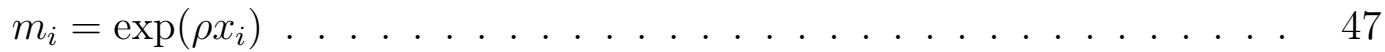

6.15 Sumário do modelo de Brody com erros skew t e função de variância

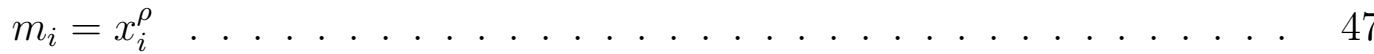

6.16 Sumário dos critérios de seleção para os modelos heteroscedásticos com erros skew-t . . . . . . . . . . . . . . . . . 47

6.17 Sumário dos critérios de seleção para o modelo com os dados perturbados 51

6.18 Identificação dos casos influentes para os dados com a perturbação . . . 53 


\section{Sumário}

1 Introdução 1

2 Modelos de crescimento $\quad 7$

2.1 Modelo de Brody . . . . . . . . . . . . . . . . . . 8

2.2 Modelo de Gompertz . . . . . . . . . . . . . . . . . . . . . . . . 9

2.3 Modelo Logístico . . . . . . . . . . . . . . . . . . . . . . . . 10

2.4 Modelo de Von Bertalanffy . . . . . . . . . . . . . . . . . . . . 11

2.5 Interpretação dos parâmetros . . . . . . . . . . . . . . . . . 11

3 Distribuições assimétricas 13

3.1 Distribuição Skew Normal . . . . . . . . . . . . . . . . . . . . 14

3.1.1 Propriedades ........................ 15

3.2 Distribuição Skew t . . . . . . . . . . . . . . . . . . . . . 16

3.2 .1 Propriedades . . . . . . . . . . . . . . . . . 18

4 Inferência bayesiana para as distribuições assimétricas $\quad 19$

4.1 Modelo Skew Normal . . . . . . . . . . . . . . . . . . . . . . . . . . . 20 
4.2 Modelo Skew t . . . . . . . . . . . . . . . . . . . 21

5 Modelos de regressão não linear com erros assimétricos 25

5.1 Caso homoscedástico . . . . . . . . . . . . . . . . . . 26

5.1 .1 Erros com distribuição skew normal . . . . . . . . . . . . 26

5.1.2 Erros com distribuição skew t . . . . . . . . . . . . . . . 27

5.2 Caso heteroscedástico . . . . . . . . . . . . . . . . . . . . . . . . . 28

5.2.1 Erros com distribuição skew normal . . . . . . . . . . . . . . 29

5.2 .2 Erros com distribuição skew t . . . . . . . . . . . . . 30

5.3 Critérios de seleção de modelos . . . . . . . . . . . . . . . . . . . . . 31

5.4 Diagnóstico . . . . . . . . . . . . . . . . . . . . . . . . . . 34

6 Aplicação $\quad 37$

6.1 Estudo com um conjunto de dados simulados . . . . . . . . . . . . . . . 37

6.2 Aplicação para um conjunto de dados . . . . . . . . . . . . . . . . . . . 39

6.2.1 Caso homocedástico . . . . . . . . . . . . . . . . . 40

6.2.2 Caso heteroscedástico . . . . . . . . . . . . . . . . . 45

6.2.3 Estudo de Robustez . . . . . . . . . . . . . . . . . . . . 50

7 Conclusões e propostas futuras $\quad 55$

$\begin{array}{ll}\text { Referências Bibliográficas } & 57\end{array}$ 


\section{Capítulo 1}

\section{Introdução}

Modelos de regressão não lineares com erros normais são usualmente aplicados em muitas áreas de estudo, tais como biologia, química, medicina e sociologia, para modelar dados simétricos em que funções não lineares de parâmetros desconhecidos são usados para explicar ou descrever o fenômeno sob estudo.

Uma séries de modelos matemáticos têm sido de grande utilidade na interpretação de dados de crescimento de plantas e animais. O critério mais popular de ajuste das funções matemáticas é a estimativa dos parâmetros através do método de GaussNewton modificado e do método dos mínimos quadrados ponderados.

Especificamente, para ajuste de curvas de peso idade de bovinos, os modelos de Von Bertalanffy, Brody, Gompertz, logístico e Richards são os mais utilizados, destacando que Brown et al. (1976) e De Paz et al. (2004) usam o método de Gauss-Newton modificado para a estimação dos parâmetros dos modelos. Para os mesmos modelos citados anteriormente, Mazzini et al. (2003) consideram a heterogeneidade das variâncias.

Forni et al. (2009) comparam as quatro primeiras funções acima para descrever as curvas de crescimento de fêmeas Nelore. Os parâmetros e os componentes de variância são estimados através de um modelo hierárquico Bayesiano, e somente o modelo de 
Von Bertalanffy é utilizado em Forni et al. (2007).

As funções sigmoidais logística, Gompertz, Richards, Schute e Stannard são comparadas por Zwietering et al. (1990) para descrever a curva de crescimento bacteriana, em que o algoritmo de Marquardt minimiza a soma de quadrados das diferenças entre o valor predito e observado.

Para dados ovinos, Rocha Sarmento et al. (2006) verificam, entre os modelos Brody, Von Bertalanffy, logístico, Gompertz e Richards, aquele que melhor descreve a curva média de crescimento de ovinos Santa Inês através do método Gauss-Newton modificado, sendo que Guedes et al. (2004) utiliza dados de cordeiros das raças Santa Inês e Bergamácia considerando o método dos mínimos quadrados ponderados pelo inverso da variância dos pesos dos animais.

Fekedulegn et al. (1999) apresentam a aplicação de derivadas parciais na estimação dos parâmetros dos modelos de crescimento não lineares monomolecular, exponencial negativo, Mitcherlich, Gompertz, logístico, Chapman-Richards, von Bertalanffy, Weibull e Richard considerando o método iterativo de Marquardt.

O crescimento e a mortalidade de uma população de peixes é discutido por Gamito (1998), que considera os modelos exponencial, logístico, parabólico, Von Bertalanffy e Gompertz. Já Santos et al. (2008) avaliam o crescimento inicial de uma população de peixe (Nile Tilapia) ajustando um modelo exponencial considerando variâncias heteroscedásticas.

Riazoshams \& Midi (2009) analisam dados de crescimento de frangos por um modelo não linear com erros heteroscedásticos.

Em oito espécies de animais, Freitas (2007) define os modelos não lineares mais adequados para estimar o crescimento corporal desses animais através do método iterativo de Gauss-Newton, que produz estimativas a partir dos mínimos quadrados ou 
de mínimos quadrados ponderados.

Entretanto, é sabido que muitos desses estudos nem sempre estão de acordo com o modelo normal devido à falta de simetria na distribuição ou presença de distribuições com caudas mais pesadas ou mais leves que a normal.

Particularmente, as estimativas dos parâmetros de um modelo normal baseado no método de máxima verossimilhança são frequentemente sensíveis a observações atípicas. Para resolver este problema, algumas propostas são discutidas na literatura, como a substituição da suposição de normalidade por uma classe mais flexível de distribuições.

Por exemplo, Cysneiros \& Vanegas (2008) desenvolveram um modelo de regressão não linear simétrico e realizaram um estudo analítico e empírico para descrever o comportamento do resíduos padronizados. Vanegas \& Cysneiros (2010) propõem procedimentos diagnósticos baseados em modelo de deleção de casos para modelos de regressão não lineares simétricos.

Outra possibilidade considerada pelos autores são os modelos de regressão com distribuições assimétricas. Mais especificamente, as distribuições skew normal e skew t na forma univariada e multivariada.

Nesse contexto, Fernández \& Steel (1998b) e Fernández \& Steel (1998a) tratam a modelagem Bayesiana para caudas pesadas e assimetria e verificam a existência da posteriori (momentos) em regressão linear. Branco \& Dey (2002) consideram um modelo de regressão linear com erros com distribuição assimétrica de caudas pesadas.

Uma representação da classe das distribuições assimétricas é fornecida em Branco \& Dey (2001) e Ferreira \& Steel (2006).

Ferreira \& Steel (2007) introduzem um método geral para a definição das distribuições assimétricas multivariadas, utilizam esta classe de distribuições em um contexto de regressão multivariada e propõem procedimentos de inferência bayesiana. 
De la Cruz \& Branco (2009) consideram uma abordagem bayesiana para o modelo de regressão não linear utilizando erros com distribuições assimétricas, as quais considera assimetria e caudas pesadas ou somente assimetria.

Essas distribuições assimétricas são flexíveis o suficiente para acomodar assimetria e/ou caudas pesadas. Embora existam outras representações para essas distribuições propostas na literatura, uma das vantagens da abordagem proposta neste trabalho, é que, por construção, a implementação Bayesiana baseada no método de Monte Carlo via cadeia de Markov é mais fácil.

Bayes \& Branco (2007) mostram que a abordagem bayesiana usando o método MCMC é uma boa alternativa para se fazer inferência sobre um paramêtro assimétrico.

Cancho et al. (2010) discutem o modelo de regressão não linear normal assimétrico seguindo uma abordagem clássica (algoritmo EM) e bayesiana (método MCMC).

Essa dissertação está dividida em sete capítulos. No segundo, serão apresentados os modelos de crescimento de Brody, Gompertz, logístico e Von Bertalanffy, bem como suas caracterizações.

No terceiro capítulo, serão exibidas algumas distribuições assimétricas. O capítulo se divide em duas partes, em que as distribuições skew normal e skew t são apresentadas e para essas distribuições são exibidas algumas propriedades e gráficos utilizando o $\mathrm{R}$ Development Core Team (2009) como suporte de programação estatística.

No capítulo 4, utilizaremos a metodologia Bayesiana usando técnicas MCMC para o modelo skew normal e skew t. Uma forma hierárquica desses dois modelos é usada a fim de facilitar a implementação.

No quinto capítulo, apresentaremos o modelo de regressão não linear com erros assimétricos (skew normal e skew t) considerando tanto a homocedasticidade quanto a heteroscedasticidade. Utilizaremos as curvas de crescimento descritas anteriormente 
para as funções não lineares do modelo. Por fim, destacamos os critérios utilizados para a seleção do melhor modelo.

No sexto capítulo, realizamos uma aplicação com um conjunto de dados reais e fazemos uma análise de um conjunto de dados simulados.

Finalmente, no último capítulo são apresentadas nossas conclusões e perpectivas futuras de trabalho. 


\section{Capítulo 2}

\section{Modelos de crescimento}

Segundo Seber \& Wild (1989), o estudo de modelos de crescimento é importante em muitas áreas de pesquisa. Na biologia, o interesse está na descrição do crescimento de animais e plantas na tentativa de entender seus mecanismos essenciais. Os químicos utilizam esses modelos na formulação de uma reação química sobre o tempo. Na agricultura, são óbvias as vantagens econômicas e administrativas em conhecer como a produção cresce, o quão rápido elas crescem, e como esses fatores são responsáveis pelas condições do meio ou do tratamento. O crescimento infantil é tema de interesse na medicina, assim como o crescimento de tumores e os efeitos dos tratamentos sobre tais crescimentos. Cientistas socias têm interesses em crescimento da população, fornecimento de alimentos e na demanda de energia.

O termo curva de crescimento usualmente evoca a imagem de curvas sigmoidais que representam o tempo de vida de medidas de dimensão, geralmente de altura e peso.

$\mathrm{Na}$ modelagem de dados de crescimento, desejamos obter informações sobre a interpretação física dos parâmetros, a fim de construir um modelo padrão para as observações em estudo.

Vale ressaltar que uma grande vantagem desses modelos é a simplicidade e facili- 
dade na interpretação dos parâmetros, pois em muitas situações, são requeridos menos parâmetros nos modelos não lineares do que nos lineares. Além disso, nos modelos não lineares, os parâmetros fornecem um maior conhecimento sobre o fenômeno em estudo, o que gera um bom ajuste, com menos parâmetros.

Para as inferências de interesse nessa classe de modelos não lineares, em geral, é considerado um processo iterativo (por exemplo, o método de Gauss Newton) para encontrar os estimadores de mínimos quadrados e a construção de testes de hipóteses ou intervalos de confiança em geral, são baseados em resultados aproximados. É comum também o uso do método de máxima verossimilhança para a estimação dos parâmetros do modelo e o uso dos resultados assintóticos usuais para os estimadores de máxima verossilhança na construção de testes de hipóteses e intervalos de confiança.

Quando esses resultados não são muitos precisos, diferentes parametrizações são utilizadas para melhorar a precisão dos resultados obtidos.

Porém, esses parâmetros possuem interpretações físicas relacionadas ao experimento e muitas vezes, o pesquisador não quer formas transformadas dos parâmetros originais.

Portanto, uma análise Bayesiana dos modelos não lineares pode ser de grande interesse e utilidade prática.

A seguir, apresentaremos os modelos utilizados neste trabalho para ajustar curvas de crescimento: modelo de Brody, modelo de Gompertz, modelo logístico e modelo de Von Bertalanffy.

\subsection{Modelo de Brody}

Brody (1945) propôs a divisão de duas curvas sigmoidais. A primeira função é 
exponencialmente crescente

$$
f(x)=\exp \left(\beta_{3} x\right)
$$

Sua assíntota é infinita, entretanto, esta curva é aplicada somente em intervalos limitados e não pode ser extrapolada.

A segunda função é exponencialmente decrescente

$$
f(x)=\beta_{1}\left(1-\beta_{2} e^{-\beta_{3} x}\right)
$$

e será adotada para as aplicações do capítulo 6.

\subsection{Modelo de Gompertz}

Este modelo foi desenvolvido por Gompertz (1825) para o cálculo da taxa de mortalidade. É um tipo de modelo matemático para uma série temporal, em que o crescimento é mais lento no início e no final de um período de tempo. O valor assintótico superior desta curva é mais gradual que o valor assintótico inferior, em contraste com a função logística em que ambas as assíntotas são simétricas. É ainda um dos modelos mais utilizados, mas por muito tempo foi de interesse somente para os atuários. Mas recentemente, entretanto, tem sido usado por vários autores como uma curva de crescimento, tanto para estudos biológicos como econômicos. O modelo de Gompertz é definido por

$$
f(x)=\beta_{1} e^{-\beta_{2} e^{-\beta_{3} x}}
$$




\subsection{Modelo Logístico}

Verhulst (1938) propôs uma função para modelar o crescimento populacional. Esta função pode ser usada também para crescimento somático e é definida por

$$
f(x)=\frac{\beta_{1}}{1+\beta_{2} e^{-\beta_{3} x}}
$$

Os pontos de inflexão são $x=\ln \frac{\beta_{2}}{\beta_{3}}$ e $f(x)=\frac{\beta_{1}}{2}$ e independem dos valores de $x$.

O modelo logístico é geralmente utilizado para crescimentos sigmoidais em que o ponto de inflexão está localizado aproximadamente na metade do último valor medido.

Essa curva descreve os processos de crescimento natural de qualquer sistema. Um processo de crescimento natural consiste em preencher um determinado nicho desde o início até a sua saturação, uma vez que todo o nicho a ser preenchido apresenta limite de capacidade. Assim, o crescimento de uma população (humana ou de qualquer espécie animal e planta), a difusão de uma epidemia ou de uma inovação tecnológica, o crescimento do mercado de um produto, o crescimento de um ser vivo ou de uma população, etc., são considerados como processos de crescimento natural e são descritos por curvas logísticas. Os processos de aprendizagem são também processos de crescimento natural, e por isso as curvas logísticas são também designadas por curvas de aprendizagem. Quando um indivíduo, ou um grupo, ou uma coletividade de pessoas aprende a realizar uma determinada tarefa ou aprende um determinado tema, o que está a acontecer é o crescimento cumulativo de informações, que começa com uma informação inicial e aumenta até o esgotamento da informação para executar a tarefa ou para dominar o tema em questão. 


\subsection{Modelo de Von Bertalanffy}

Bertalanffy (1957) apresentou uma função para o crescimento do peso corporal, definida por

$$
f(x)=\beta_{1}\left(1-\beta_{2} e^{-\beta_{3} x}\right)^{3}
$$

O modelo acima ajusta processos de crescimento sigmoidais, no qual o ponto de inflexão está localizado aproximadamente em $30 \%$ do último valor medido.

\subsection{Interpretação dos parâmetros}

Os modelos de crescimento apresentados anteriormente, possuem a seguinte interpretação para seus parâmetros. O parâmetro $\beta_{1}$ representa o valor assintótico da variável resposta, $\beta_{2}$ é uma constante que está relacionada ao valor observado inicial, indicando a proporção do valor assintótico a ser ganho. O parâmetro $\beta_{3}$ é a taxa de crescimento da variável resposta, indica a velocidade com que o valor observado se aproxima do valor máximo, determina a eficiência do crescimento.

A figura 2.5 apresenta o gráfico dos modelos citados anteriormente para os seguintes valores dos parâmetros: $\beta_{1}=1, \beta_{2}=1, \beta_{3}=1$. 


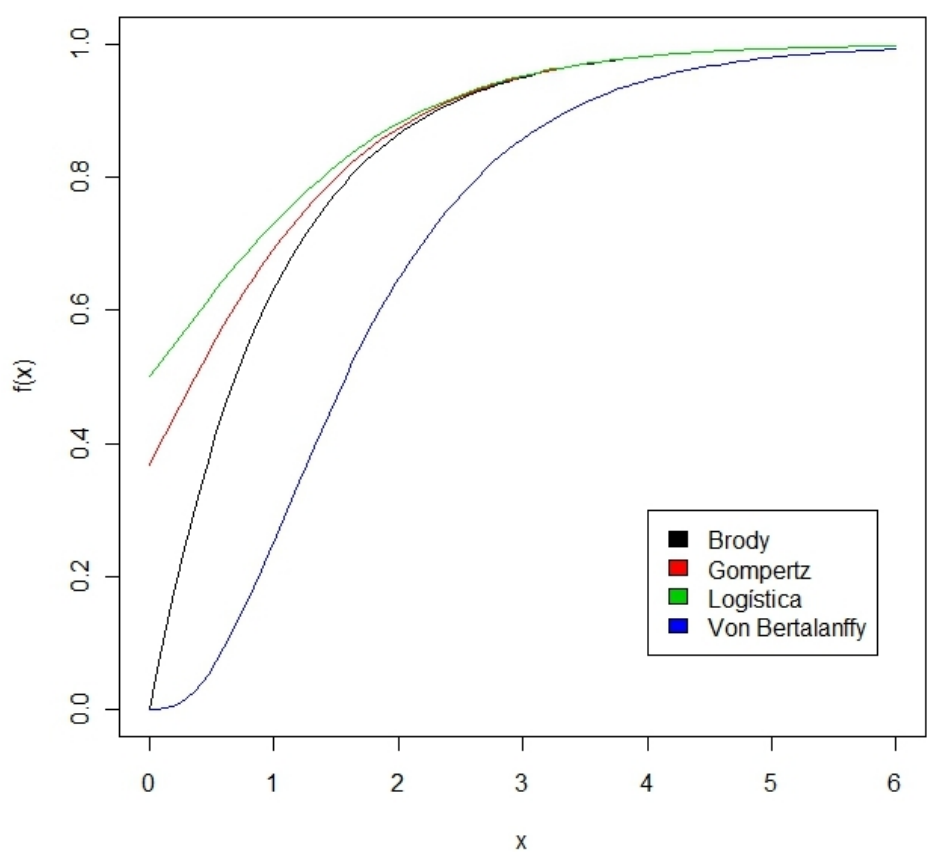

Figura 2.1: Curvas de crescimento. 


\section{Capítulo 3}

\section{Distribuições assimétricas}

O estudo de variáveis aleatórias contínuas desenvolvido em grande parte da análise estatística, está baseado no modelo normal. Entretanto, ao modelar um conjunto de dados considerando a distribuição normal, deve-se assegurar a suposição de simetria. Se esta condição for violada, as inferências obtidas ao se supor normalidade podem ficar prejudicadas.

Outras propostas de modelagem têm sido discutidas a fim de minimizar este problema. Uma das alternativas, discutida por Arellano-Valle (1994) é utilizar a distribuição t-Student que reduz a influência de valores extremos nas inferências, sendo considerado um modelo mais robusto que o normal (Lange et al. (1989)).

Porém, tanto a distribuição normal quanto a distribuição t-Student pressupõem a simetria dos dados. Assim, seria interessante obter distribuições assimétricas que tenham como caso particular distribuições simétricas conhecidas, como é o caso da distribuição skew normal e skew t.

A distribuição skew normal foi introduzida formalmente por Azzalini (1985), que estudou suas propriedades e verificou que esta distribuição tem problemas na estimação do parâmetro que controla a assimetria, pelos métodos dos momentos e de máxima 
verossimilhança. Várias propostas para o caso multivariado podem ser encontrados em Azzalini \& Dalla Valle (1996), Azzalini \& Capitanio (1999), Sahu et al. (2003) e Genton et al. (2001).

A distribuição skew t é uma distribuição assimétrica importante pois inclui não só a distribuição t-Student como caso particular, mas também as distribuições normal e skew normal em casos limites. Os primeiros trabalhos que apresentaram esta distribuição foram Branco \& Dey (2001) e Azzalini \& Capitanio (2003).

Neste capítulo faremos uma revisão sobre a caracterização e algumas propriedades das distribuições skew normal e skew t.

\subsection{Distribuição Skew Normal}

A distribuição skew normal representa a generalização da distribuição normal na qual tem um parâmetro de forma adicional que define a direção da assimetria da distribuição.

Como considerado em Azzalini (1985), uma variável aleatória $Z$ tem distribuição skew normal com parâmetro de locação $\mu$, parâmetro de escala $\sigma^{2}$ e parâmetro de assimetria $\lambda$, se sua função de densidade for dada por

$$
f(z)=2 \phi\left(z ; \mu, \sigma^{2}\right) \Phi\left(\frac{\lambda(z-\mu)}{\sigma}\right)
$$

em que $\phi\left(\cdot ; \mu, \sigma^{2}\right)$ denota a densidade de uma distribuição normal univariada com média $\mu$ e variância $\sigma^{2}>0$ e $\Phi(\cdot)$ a função distribuição de uma normal univariada padrão. Valores negativos de $\lambda$ indicam assimetria negativa e valores positivos de $\lambda$ assimetria positiva. Se $\lambda=0$ a densidade acima coincide com a densidade da distribuição normal e

portanto é simétrica. Utilizaremos a notação $Z \sim S N(\mu, \sigma, \lambda)$ para denotar esta classe 
de distribuições. Note que esta densidade pode ser bastante assimétrica dependendo da escolha do parâmetro $\lambda$, como ilustrado na Figura 3.1.

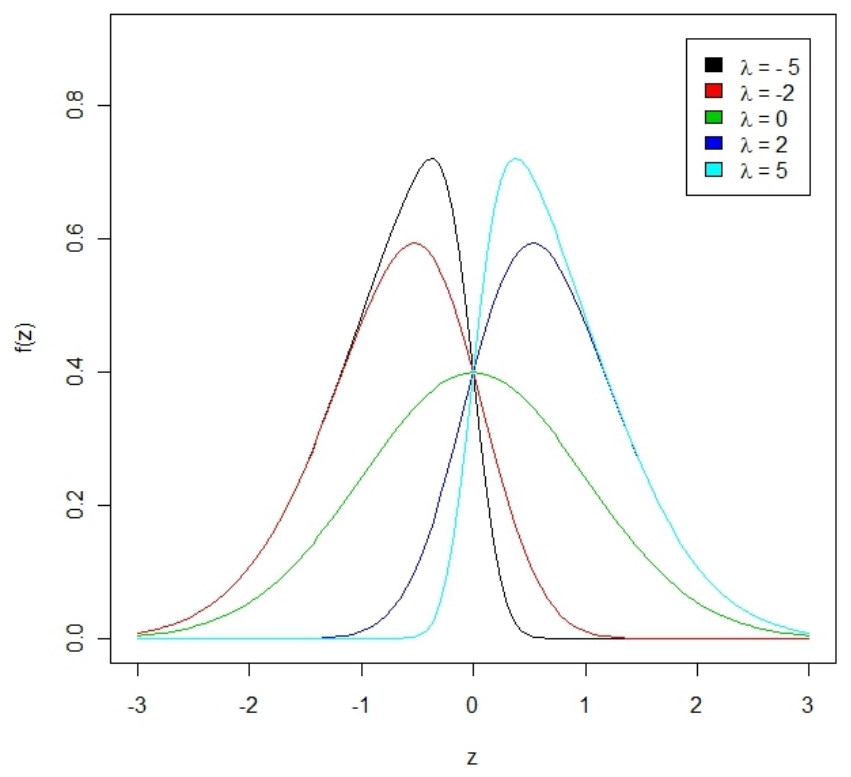

Figura 3.1: Funções de densidade para a distribuição skew normal padrão, com diferentes valores para $\lambda$.

Uma forma de apresentar a distribuição skew normal é através da representação estocástica, à qual foi obtida por Henze (1986) e cujo resultado é dado abaixo

$$
Z=\mu+\sigma\left(\delta\left|T_{0}\right|+\left(1-\delta^{2}\right)^{1 / 2} T_{1}\right), \operatorname{com} \delta=\frac{\lambda}{\sqrt{1+\lambda^{2}}}
$$

em que $T_{0}$ e $T_{1}$ são variáveis independentes com distribuição normal padrão.

\subsubsection{Propriedades}

A densidade em (3.1) possui algumas propriedades interessantes que serão apenas listadas neste trabalho e cujas provas podem ser obtidas em Azzalini (1985). 
1. A função geradora de momentos de $Z$ é dada por

$$
M(t)=2 \exp \left(\frac{(t-\mu)^{2}}{2 \sigma^{2}}\right) \Phi\left(\delta \frac{t-\mu}{\sigma}\right)
$$

2. $E(Z)=\mu+\sqrt{\frac{2}{\pi}} \sigma \delta$

3. $\operatorname{Var}(Z)=\sigma^{2}\left(1-\frac{2}{\pi} \delta^{2}\right)$

4. $E\left(Z^{3}\right)=\mu^{3}+3 \mu^{3} \sigma \delta \sqrt{\frac{2}{\pi}}+3 \mu \sigma^{2}+3 \sigma^{3} \delta \sqrt{\frac{2}{\pi}}-\sigma^{3} \delta^{3} \sqrt{\frac{2}{\pi}}$

5. $\gamma=\sqrt{\frac{2}{\pi}} \delta^{3}\left[\frac{4}{\pi}-1\right]\left[1-\frac{2 \delta^{2}}{\pi}\right]^{-3 / 2}$

em que $\gamma$ é o coeficiente de assimetria com $-0,99527<\gamma<0,99527$ e $\delta=\frac{\lambda}{\sqrt{1+\lambda^{2}}}$. Esta medida caracteriza como e quanto a distribuição se afasta da condição de simetria.

\subsection{Distribuição Skew t}

Uma variável aleatória Y segue uma distribuição skew t, com parâmetro de locação $\mu$, parâmetro de escala $\sigma^{2}$, parâmetro de curtose $\nu>0$ e parâmetro de assimetria $\lambda$ se sua função densidade de probabilidade é dada por

$$
f(y)=\frac{\Gamma\left(\frac{\nu+1}{2}\right)}{\Gamma\left(\frac{\nu}{2}\right) \sqrt{\pi \nu \sigma}}\left(1+\frac{d}{\nu}\right)^{-\frac{\nu+1}{2}} T\left(\sqrt{\frac{\nu+1}{d+\nu}} A ; \nu+1\right), y \in \mathbb{R}
$$

em que $d=(y-\mu)^{2} / \sigma^{2}, A=\frac{\lambda(y-\mu)}{\sigma}$ e $T(\cdot ; \nu)$ representa a função distribuição acumulada da distribuição t-Student padrão, com locação zero, escala um e $\nu$ graus de liberdade, denotada por $t(0,1, \nu)$. O parâmetro $\lambda$ regula a forma da distribuição skew t. Valores negativos de $\lambda$ indicam assimetria negativa e valores positivos de $\lambda$ indicam assimetria positiva. Quando $\lambda=0$, a densidade definida anteriormente será simétrica e irá coincidir com a densidade da distribuição $t$. 
Usaremos a notação $Y \sim S T\left(\mu, \sigma^{2}, \lambda, \nu\right)$. Na Figura (3.2) apresentamos alguns gráficos que ilustram o comportamento da densidade descrita em (3.3) para $\mu=0$ e $\sigma^{2}=1$ fixados, para $\lambda$ igual a $0,2,10$ e 50, fixado o valor de $\nu$, e para valores de $\nu$ iguais a 2,5 e 15 , fixado o valor de $\lambda$.

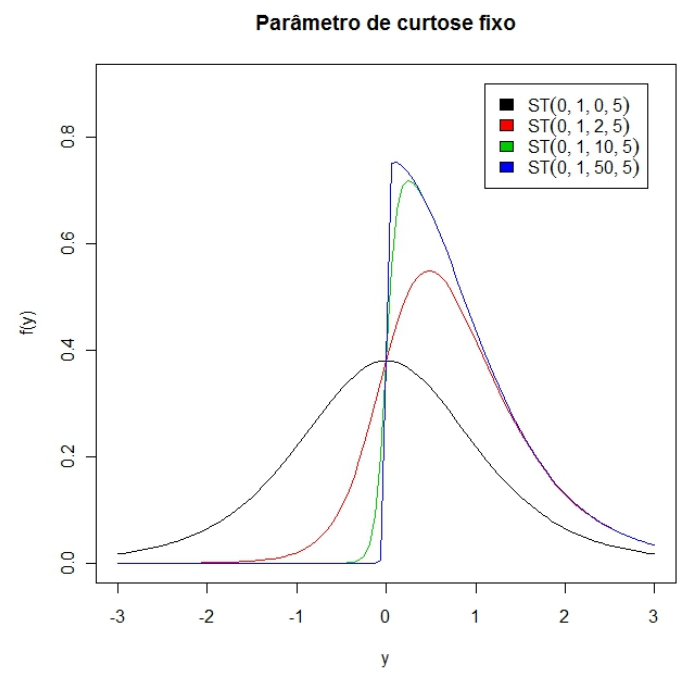

(a) $\nu=5$

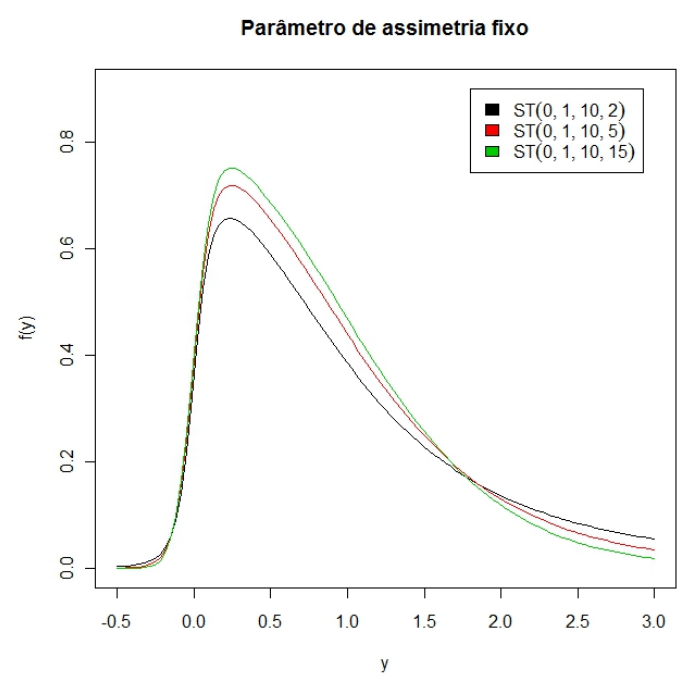

(b) $\lambda=10$

Figura 3.2: Funções de densidade para a distribuição skew t padrão, com diferentes valores para $\lambda$ e $\nu$.

Apresentamos a seguir uma maneira de obter a distribuição skew t, que consiste em obter $Y$ como combinação de variáveis aleatórias independentes.

$$
Y=\mu+U^{-1 / 2} Z
$$

em que $Z \sim S N\left(0, \sigma^{2}, \lambda\right)$ e $U \sim \operatorname{Gama}(\nu / 2, \nu / 2)$ com função densidade de probabilidade da forma

$$
h(u \mid \nu)=\frac{(\nu / 2)^{\nu / 2} u^{\nu / 2-1}}{\Gamma(\nu / 2)} \exp \left(-\frac{1}{2} \nu u\right)
$$

independente de $Z$ (Gupta (2003)).

De (3.2), segue que (3.4) pode ser escrito como 


$$
Y=\mu+\Delta T+U^{-1 / 2} \tau^{1 / 2} T_{1}
$$

em que $\Delta=\sigma \delta, \delta=\frac{\lambda}{\sqrt{1+\lambda^{2}}}, \tau=\left(1-\delta^{2}\right) \sigma^{2}, T=U^{-1 / 2}\left|T_{0}\right|, T_{0}$ e $T_{1}$ são distribuições normais padrão independentes e $|\cdot|$ denota o valor absoluto.

Algumas propriedades da distribuição skew t são descritas abaixo.

\subsubsection{Propriedades}

1. A função geradora de momentos de $Z$ é dada por

$$
M(t)=e^{t \mu} \int_{0}^{\infty} M_{Z}\left(\frac{t \sigma}{\sqrt{w}}\right) d w
$$

em que $h(w)$ corresponde à função densidade de probabilidade de uma distribuição Gama $\left(\frac{\nu}{2}, \frac{\nu}{2}\right)$ e $M_{Z}(t)$ corresponde à função geradora de momentos da distribuição skew normal padrão.

2. $E(Y)=\mu+\sqrt{\frac{2}{\pi}} k_{1} \Delta$

3. $\operatorname{Var}(Y)=\sigma^{2}\left(k_{2}-b^{2} \delta^{2}\right)$ em que $b=-\sqrt{\frac{2}{\pi}}$ e $k_{m}=\left(\frac{\nu}{2}\right)^{m} / 2 \frac{\Gamma\left(\frac{\nu-m}{2}\right)}{\Gamma\left(\frac{\nu}{2}\right)}$.

4. Coeficiente de assimetria

$$
\gamma=\beta\left[\left(3-\delta^{2}\right) \frac{\nu}{\nu-3}-3 \frac{\nu}{\nu-2}+2 \beta^{2}\right]\left[\frac{\nu}{\nu-2}-\beta^{2}\right]^{-\frac{3}{2}}, \text { para } \nu>3
$$

em que $\beta=E(Y)$ 


\section{Capítulo 4}

\section{Inferência bayesiana para as}

\section{distribuições assimétricas}

A inferência nos modelos assimétricos ainda é objeto de investigação. As abordagens pelo método clássico são as que apresentam mais problemas de identificabilidade, estimação e convergência, tanto pelo método dos momentos como no de máxima verossimilhança. Tais dificuldades estão relacionadas essencialmente ao fato de que o conjunto de valores admissíveis para o coeficiente de assimetria é limitado.

A abordagem bayesiana tem apresentado melhores resultados mesmo quando a priori não é informativa. Esta abordagem é de fácil implementação em softwares como R Development Core Team (2009) e WinBUGS (Spiegelhalter et al. (2003), Lunn et al. (2000)), por exemplo. Na linguagem BUGS as gerações das cadeias de Markov já estão prontas.

Neste capítulo é discutido o método de inferência bayesiana para os parâmetros dos modelos skew normal e skew t. 


\subsection{Modelo Skew Normal}

Nesta seção, desenvolveremos a metodologia Bayesiana usando técnicas MCMC para o modelo skew normal. Uma forma hierárquica de reescrever este modelo é utilizando a representação estocástica de Henze (1986), dado por (3.2). Dessa forma, obtemos

$$
\begin{gathered}
Y_{i} \mid T_{i}=t_{i} \sim N\left(\mu+\sigma \delta t_{i}, \sigma^{2}\left(1-\delta^{2}\right)\right), \\
T_{i} \sim H N(0,1),
\end{gathered}
$$

em que $H N(0,1)$ é a distribuição meia normal padrão.

Para facilitar o trabalho computacional, foi considerado a seguinte reparametrização $\Delta=\sigma \delta$ e $\tau=\left(1-\delta^{2}\right) \sigma^{2}$, então

$$
\begin{gathered}
Y_{i} \mid T_{i}=t_{i} \sim N\left(\mu+\Delta t_{i}, \tau\right), \\
T_{i} \sim H N(0,1) .
\end{gathered}
$$

Seja $\boldsymbol{y}=\left(y_{1}, \ldots, y_{n}\right)^{T}$ e $\boldsymbol{t}=\left(t_{1}, \ldots, t_{n}\right)^{T}$. Segue que a função de verossimilhança para $\boldsymbol{\theta}=\left(\mu, \sigma^{2}, \lambda\right)^{T}$ é dada por

$$
L(\boldsymbol{\theta})=\frac{1}{\tau^{n / 2}} \exp \left(-\frac{1}{2 \tau} \sum_{i=1}^{n}\left(y_{i}-\mu-\Delta t_{i}\right)^{2}\right) \exp \left(-\frac{1}{2} \sum_{i=1}^{n} t_{i}^{2}\right) \prod_{i=1}^{n} I_{\left\{t_{i}>0\right\}}
$$

Os parâmetros foram considerados independentes e com as seguintes distribuições a priori

$$
\mu \sim N\left(a, b^{2}\right)
$$




$$
\begin{aligned}
& \sigma^{2} \sim I G(u, s) \\
& \lambda \sim N\left(\mu_{\lambda}, \sigma_{\lambda}^{2}\right)
\end{aligned}
$$

tomando $\delta=\frac{\lambda}{\sqrt{1+\lambda^{2}}}$.

A densidade a posteriori é dada por

$$
\begin{aligned}
\pi\left(\mu, \sigma^{2}, \lambda \mid \boldsymbol{z}, \boldsymbol{t}\right) & \propto \frac{1}{\tau^{n / 2}}\left(\sigma^{2}\right)^{-(u+1)} \exp \left(-\frac{1}{2} \sum_{i=1}^{n} t_{i}^{2}\right) \prod_{i=1}^{n} I_{\left\{t_{i}>0\right\}} \\
& \times \exp \left[-\frac{1}{2 \tau} \sum_{i=1}^{n}\left(y_{i}-\mu-\Delta t_{i}\right)^{2}-\frac{(\mu-a)^{2}}{2 b^{2}}-\frac{\left(\lambda-\mu_{\lambda}\right)^{2}}{2 \sigma_{\lambda}^{2}}-\frac{s}{\sigma^{2}}\right] .
\end{aligned}
$$

Assim, as densidades a posteriori marginais para os três parâmetros são dadas por

$$
\begin{gathered}
\pi\left(\mu \mid \sigma^{2}, \lambda, \boldsymbol{z}, \boldsymbol{t}\right) \propto \exp \left[-\frac{1}{2 \tau} \sum_{i=1}^{n}\left(y_{i}-\mu-\Delta t_{i}\right)^{2}-\frac{1}{2 b^{2}}(\mu-a)^{2}\right] \\
\pi\left(\sigma^{2} \mid \mu, \lambda, \boldsymbol{z}, \boldsymbol{t}\right) \propto \frac{1}{\tau^{n / 2}}\left(\sigma^{2}\right)^{-(u+1)} \exp \left[-\frac{1}{2 \tau} \sum_{i=1}^{n}\left(y_{i}-\mu-\Delta t_{i}\right)^{2}-\frac{s}{\sigma^{2}}\right]
\end{gathered}
$$

$\mathrm{e}$

$$
\pi\left(\lambda \mid \mu, \sigma^{2}, \boldsymbol{z}, \boldsymbol{t}\right) \propto \frac{1}{\tau^{n / 2}} \exp \left[-\frac{1}{2 \tau} \sum_{i=1}^{n}\left(y_{i}-\mu-\Delta t_{i}\right)^{2}-\frac{1}{2 \sigma_{\lambda}^{2}}\left(\lambda-\mu_{\lambda}\right)^{2}\right] .
$$

\subsection{Modelo Skew t}

Nesta seção, desenvolveremos a metodologia Bayesiana usando técnicas MCMC para o modelo skew t. Uma forma hierárquica de reescrever este modelo é utilizando a representação estocástica dada por (3.4). Dessa forma, obtemos 


$$
\begin{aligned}
Y_{i} \mid T_{i}=t_{i} \sim N\left(\mu+\sigma \delta t_{i}, \frac{1}{U_{i}} \sigma^{2}\left(1-\delta^{2}\right)\right), \\
T_{i} \mid U_{i} \sim T N\left(n, u_{i}^{-1}\right) I_{\{n, \infty\}}, \\
U_{i} \sim \operatorname{Gama}\left(\frac{\nu}{2}, \frac{\nu}{2}\right),
\end{aligned}
$$

em que $T N(r, s) I_{\{a, b\}}$ denota a distribuição normal truncada em $(a, b)$, com média $a$ e variância $b$ antes do truncamento.

Para facilitar o trabalho computacional, foi considerado a seguinte reparametrização $\Delta=\sigma \delta$ e $\tau=\left(1-\delta^{2}\right) \sigma^{2}$, então

$$
\begin{gathered}
Y_{i} \mid T_{i}=t_{i} \sim N\left(\mu+\Delta t_{i}, \frac{1}{U_{i}} \tau\right), \\
T_{i} \mid U_{i} \sim T N\left(n, u_{i}^{-1}\right) I_{\{n, \infty\}}, \\
U_{i} \sim \operatorname{Gama}\left(\frac{\nu}{2}, \frac{\nu}{2}\right) .
\end{gathered}
$$

Seja $\boldsymbol{y}=\left(y_{1}, \ldots, y_{n}\right)^{T}$ e $\boldsymbol{t}=\left(t_{1}, \ldots, t_{n}\right)^{T}$ e $\boldsymbol{u}=\left(u_{1}, \ldots, u_{n}\right)^{T}$. Segue que a função de verossimilhança para $\boldsymbol{\theta}=\left(\mu, \sigma^{2}, \lambda, \nu\right)^{T}$ é dada por

$$
\begin{aligned}
L(\boldsymbol{\theta}) & =\prod_{i=1}^{n}\left(\frac{1}{u_{i}^{-1} \tau}\right) \exp \left(-\sum_{i=1}^{n} \frac{\left(y_{i}-\mu-u_{i}^{-\frac{1}{2}} \Delta t_{i}\right)^{2}}{2 \tau u_{i}^{-1}}\right) \prod_{i=1}^{n}\left(\frac{1}{u_{i}^{-1}}\right) \\
& \times \exp \left(-\sum_{i=1}^{n} \frac{\left(t_{i}-n\right)^{2}}{2 u_{i}^{-1}}\right) \prod_{i=1}^{n} I_{\{n>0\}} \prod_{i=1}^{n}\left(\frac{(\nu / 2)^{\nu / 2} u_{i}^{\nu / 2-1}}{\Gamma(\nu / 2)}\right) \exp \left(-\frac{1}{2} \sum_{i=1}^{n} \nu u_{i}\right)
\end{aligned}
$$

Para completar a especificação Bayesiana do modelo foi considerado as seguintes distribuições a priori para os parâmetros desconhecidos $\boldsymbol{\theta}$.

$$
\mu \sim N\left(a, b^{2}\right)
$$




$$
\begin{gathered}
\sigma^{2} \sim I G(u, s) \\
\lambda \sim N\left(\mu_{\lambda}, \sigma_{\lambda}^{2}\right) \\
\nu \sim \operatorname{Exp}(d) I_{\{\nu>2\}}
\end{gathered}
$$

tomando $\delta=\frac{\lambda}{\sqrt{1+\lambda^{2}}}$.

A densidade a posteriori é dada por

$$
\begin{aligned}
\pi\left(\mu, \sigma^{2}, \lambda, \nu \mid \boldsymbol{y}, \boldsymbol{t}, \boldsymbol{u}\right) & \propto \prod_{i=1}^{n}\left(\frac{(\nu / 2)^{\nu / 2} u_{i}^{\nu / 2-1}}{u_{i}^{-1} \tau \Gamma(\nu / 2)}\right)\left(\sigma^{2}\right)^{-(u+1)} \\
& \times \prod_{i=1}^{n}\left(\frac{1}{u_{i}^{-1}}\right) \exp \left(-\sum_{i=1}^{n} \frac{\left(t_{i}-n\right)^{2}}{2 u_{i}^{-1}}\right) \prod_{i=1}^{n} I_{\{n>0\}} \\
& \times \exp \left[-\frac{1}{2} \sum_{i=1}^{n}\left(\frac{\left(y_{i}-\mu-u_{i}^{-\frac{1}{2}} \Delta t_{i}\right)^{2}}{\tau u_{i}^{-1}}+\nu u_{i}\right)\right] \\
& \times \exp \left(-\frac{(\mu-a)^{2}}{2 b^{2}}-\frac{\left(\lambda-\mu_{\lambda}\right)^{2}}{2 \sigma_{\lambda}^{2}}-\frac{s}{\sigma^{2}}-d \nu\right) I_{\{\nu>2\} .} .
\end{aligned}
$$

Assim, as densidades a posteriori marginais para os quatro parâmetros são dadas por

$$
\begin{gathered}
\pi\left(\mu \mid \sigma^{2}, \lambda, \nu, \boldsymbol{y}, \boldsymbol{t}, \boldsymbol{u}\right) \propto \exp \left[-\frac{1}{2 \tau} \sum_{i=1}^{n} \frac{\left(y_{i}-\mu-u_{i}^{-\frac{1}{2}} \Delta t_{i}\right)^{2}}{u_{i}^{-1}}-\frac{(\mu-a)^{2}}{2 b^{2}}\right], \\
\pi\left(\sigma^{2} \mid \mu, \lambda, \nu, \boldsymbol{y}, \boldsymbol{t}, \boldsymbol{u}\right) \propto \prod_{i=1}^{n}\left(\frac{1}{u_{i}^{-1} \tau}\right)\left(\sigma^{2}\right)^{-(u+1)} \exp \left[-\sum_{i=1}^{n} \frac{\left(y_{i}-\mu-u_{i}^{-\frac{1}{2}} \Delta t_{i}\right)^{2}}{2 \tau u_{i}^{-1}}-\frac{s}{\sigma^{2}}\right], \\
\pi\left(\lambda \mid \mu, \sigma^{2}, \nu, \boldsymbol{y}, \boldsymbol{t}, \boldsymbol{u}\right) \propto \prod_{i=1}^{n}\left(\frac{1}{u_{i}^{-1} \tau}\right) \exp \left[-\sum_{i=1}^{n} \frac{\left(y_{i}-\mu-u_{i}^{-\frac{1}{2}} \Delta t_{i}\right)^{2}}{2 \tau u_{i}^{-1}}-\frac{\left(\lambda-\mu_{\lambda}\right)^{2}}{2 \sigma_{\lambda}^{2}}\right],
\end{gathered}
$$

$\mathrm{e}$

$$
\pi\left(\nu \mid \mu, \sigma^{2}, \lambda, \boldsymbol{y}, \boldsymbol{t}, \boldsymbol{u}\right) \propto \prod_{i=1}^{n}\left(\frac{(\nu / 2)^{\nu / 2} u_{i}^{\nu / 2-1}}{\Gamma(\nu / 2)}\right) \exp \left(-\frac{\nu}{2} \sum_{i=1}^{n} u_{i}-d \nu\right) I_{\{\nu>2\}}
$$

Todas as representações hierárquicas dos modelos skew normal e skew t e respectivas 
especificações a priori são facilmente implementadas no software WinBUGS, que utiliza o Algoritmo de Gibbs, e variações deste, para obter amostras da distribuição a posteriori e a partir delas obter medidas de interesse das distribuições a posteriori marginais. 


\section{Capítulo 5}

\section{Modelos de regressão não linear com erros assimétricos}

Em muitas áreas tais como finanças, economia, sociologia, climatologia, engenharia e biologia, algumas vezes a estrutura dos erros nos modelos de regressão não satisfaz a propriedade simétrica e não se ajusta a um conjunto de dados reais. Geralmente, há presença de assimetria e caudas pesadas e a suposição de normalidade pode levar a inferências inapropriadas sobre os parâmetros de interesse e a falta de robustez.

A abordagem mais frequente para resolver essa questão é transformação de variável. Entretanto, a escolha arbitrária da transformação para simetria pode ser um problema. Em diversas situações, é difícil encontrar uma boa transformação. Até mesmo quando se obtém, o pesquisador se depara com uma nova interpretação dos parâmetros do modelo, agora como função desta nova variável resposta.

A literatura recente tem trabalhado com a variável original e propõe uma distribuição mais flexível para os erros que podem ser mais realista que a suposição de normalidade, como por exemplo Azzalini \& Capitanio (1999), DiCiccio \& Monti (2004) e Genton (2004). Nesta linha, Sahu et al. (2003) propuseram uma família assimétrica 
multivariada de distribuições com aplicação em modelo de regressão.

Do mesmo modo, a construção de flexíveis distribuições não-normal paramétricas tem recebido considerável atenção recentemente. Os principais estudos podem ser encontrados em Azzalini (1985, 2005), Gupta \& Chen (2001), Arellano-Valle \& Gomez (2004), Gomez et al. (2007), Cabral et al. (2008), entre outros.

Recentemente, Cancho et al. (2010) substituiu a suposição de normalidade dos erros em modelos de regressão usando a distribuição skew normal introduzida por Sahu et al. (2003).

Neste capítulo, apresentaremos o modelo de regressão não linear com erros assimétricos (skew normal e skew t) considerando tanto a homoscedasticidade quanto a heteroscedasticidade. Utilizaremos as curvas de crescimento descritas no capítulo 2 para as funções não lineares do modelo.

\subsection{Caso homoscedástico}

\subsubsection{Erros com distribuição skew normal}

O modelo não linear com erros seguindo a distribuição skew normal (SN) é definido por

$$
Y_{i}=\eta\left(\boldsymbol{\beta}, x_{i}\right)+\epsilon_{i}, i=1, \ldots, n .
$$

em que $Y_{i}$ é a variável resposta, $\eta(\cdot)$ é uma função injetora, contínua e duas vezes diferenciável com respeito ao vetor de parâmetros $\boldsymbol{\beta}=\left(\beta_{1}, \ldots, \beta_{p}\right)^{T}, x_{i}$ é o vetor de valores

da variável explicativa e os erros aleatórios $\epsilon_{i} \sim S N\left(-\sqrt{\frac{2}{\pi}} \Delta, \sigma^{2}, \lambda\right)$ correspondem ao modelo de regressão em que a distribuição do erro tem média zero. Assim, 


$$
E\left[Y_{i}\right]=\eta\left(\boldsymbol{\beta}, \boldsymbol{x}_{\boldsymbol{i}}\right), \operatorname{Var}\left[Y_{i}\right]=\sigma^{2}\left(1-\frac{2}{\pi} \delta^{2}\right)
$$

e $Y_{i} \sim S N\left(\eta\left(\boldsymbol{\beta}, \boldsymbol{x}_{\boldsymbol{i}}\right)-\sqrt{\frac{2}{\pi}} \Delta, \sigma^{2}, \lambda\right), i=1, \ldots, n$

Segue que a função $\log$-verossimilhança para $\boldsymbol{\theta}=\left(\boldsymbol{\beta}^{T}, \sigma^{2}, \lambda\right)^{T}$, dada pela amostra observada $\boldsymbol{y}=\left(y_{1}, \ldots, y_{n}\right)^{T}$ é dada por

$$
l(\boldsymbol{\theta})=n \log 2-\frac{n}{2} \log 2 \pi \sigma-\frac{1}{2} \sum_{i=1}^{n} B_{i}^{2}+\sum_{i=1}^{n} \log \Phi\left(\lambda B_{i}\right)
$$

em que $B_{i}=\frac{y_{i}-\eta\left(\boldsymbol{\beta}, \boldsymbol{x}_{\boldsymbol{i}}\right)+\sqrt{\frac{2}{\pi}} \Delta}{\sigma}$

\subsubsection{Erros com distribuição skew t}

O modelo de regressão não linear baseado na distribuição skew t (ST) é definido por

$$
Y_{i}=\eta\left(\boldsymbol{\beta}, x_{i}\right)+\epsilon_{i}, i=1, \ldots, n
$$

em que $Y_{i}$ é a variável resposta, $\eta(\cdot)$ é uma função injetora, contínua e duas vezes diferenciável com respeito ao vetor de parâmetros $\boldsymbol{\beta}=\left(\beta_{1}, \ldots, \beta_{p}\right)^{T}, x_{i}$ é um vetor de variáveis explicativas e erros aleatórios $\epsilon_{i} \sim S N\left(-\sqrt{\frac{2}{\pi}} k_{1} \Delta, \sigma^{2}, \lambda, H\right)$.

Para o modelo (5.2), temos que

$$
E\left[Y_{i}\right]=\eta\left(\boldsymbol{\beta}, x_{i}\right), \operatorname{Var}\left[Y_{i}\right]=\sigma^{2}\left(k_{2}-b^{2} \delta^{2}\right),
$$

em que $b=-\sqrt{\frac{2}{\pi}} k_{1}$ e $Y_{i} \sim S T\left(\eta\left(\boldsymbol{\beta}, x_{i}\right)+b \Delta, \sigma^{2}, \lambda, \nu\right)$, para $i=1, \ldots, n$.

Segue que a função log-verossimilhança para $\boldsymbol{\theta}=\left(\boldsymbol{\beta}^{T}, \sigma^{2}, \lambda, \nu^{T}\right)^{T}$, dada pela amostra 
observada $\boldsymbol{y}=\left(y_{1}, \ldots, y_{n}\right)^{T}$ é dada por

$$
l(\boldsymbol{\theta})=n \log \left(\frac{\nu+1}{2}\right)-n \log \frac{\Gamma(\nu)}{2}-\frac{n}{2} \log \pi \nu \sigma^{2}-\frac{\nu+1}{2} \sum_{i=1}^{n} \log \left(1+d_{i}\right) \sum_{i=1}^{n} \log K_{i},
$$

em que $K_{i}=T\left(\sqrt{\frac{\nu+1}{d_{i}+\nu}} A_{i} ; \nu+1\right)$ e $d_{i}=\left(y_{i}-\eta\left(\beta, x_{i}\right)+b \Delta\right) / \sigma^{2}$ e $A_{i}=d_{i}^{1 / 2} \lambda$.

\subsection{Caso heteroscedástico}

Um problema interessante a ser estudado é a heteroscedasticidade nos modelos de regressão não linear. Geralmente, a suposição padrão é considerar que todas as observações têm variâncias iguais. Porém, a violação desta suposição pode ter consequências adversas para a eficiência dos estimadores, e por esta razão, é importante detectar a heterogeneidade da variância nos modelos de regressão não lineares.

Muitos autores discutiram diversas formas para testar a heterogeneidade da variância em vários modelos. Cook \& Weisberg (1983) utilizaram o teste Score para heteroscedasticidade. Tsai (1986) propôs o teste Score para testes simultâneos de independência e homoscedasticidade em modelos lineares autoregressivos de primeira ordem. Simonoff \& Tsai (1994) propuseram testes diagnósticos para variância não constante em modelos lineares. Diblasi \& Bowman (1997) construíram um teste não paramétrico de variância constante em modelos lineares. Lin et al. (2004, 2009), respectivamente, consideraram heteroscedasticidade e verificaram mudanças na dispersão nos modelos de regressão t e Gaussiana Inversa. Xie et al. (2009) investigou o teste Score para modelos de regressão não linear skew normal.

Aqui, para a estrutura heteroscedástica, tanto a média como o parâmetro de dispersão variam com as observações através de funções não lineares. Em Davidian \& 
Carrol (1987), várias funções para a variância de modelos não lineares são sugeridas, e uma forma mais geral é recomendada. Vamos considerar algumas funções que são casos particulares da forma geral sugerida.

\subsubsection{Erros com distribuição skew normal}

O modelo de regressão não linear com erros seguindo a distribuição skew normal (ST) é definido por

$$
Y_{i}=\eta\left(\boldsymbol{\beta}, x_{i}\right)+\epsilon_{i}, i=1, \ldots, n,
$$

em que $y_{i}$ é a variável resposta, $\eta(\cdot)$ é uma função injetora, contínua e duas vezes diferenciável com respeito ao vetor de parâmetros $\boldsymbol{\beta}=\left(\beta_{1}, \ldots, \beta_{p}\right)^{T}, x_{i}$ é um vetor de variáveis explicativas e erros aleatórios $\epsilon_{i} \sim S N\left(-\sqrt{\frac{2}{\pi}} \Delta m_{i}^{1 / 2}, m_{i} \sigma^{2}, \lambda\right)$, em que $m_{i}=$ $m\left(\rho, x_{i}\right)$ é uma função conhecida, diferenciável, contínua e positiva e $\boldsymbol{x}_{i}=\left(x_{i 1}, \ldots, x_{i q}\right)^{T}$ são valores da variável explicativa e $\rho$ um parâmetro desconhecido.

Para o modelo (5.3), temos que

$$
E\left[Y_{i}\right]=\eta\left(\boldsymbol{\beta}, x_{i}\right), \operatorname{Var}\left[Y_{i}\right]=m_{i} \sigma^{2}\left(1-\frac{2}{\pi} \delta^{2}\right)
$$

em que $b=-\sqrt{\frac{2}{\pi}}$ e $Y_{i} \sim S N\left(\eta\left(\beta, x_{i}\right)+b \Delta m_{i}^{1 / 2}, m_{i} \sigma^{2}, \lambda\right)$, para $i=1, \ldots, n$.

Segue que a função log-verossimilhança para $\boldsymbol{\theta}=\left(\boldsymbol{\beta}^{T}, \sigma^{2}, \lambda\right)^{T}$, dada pela amostra observada $\boldsymbol{y}=\left(y_{1}, \ldots, y_{n}\right)^{T}$ é dada por

$$
l(\boldsymbol{\theta})=n \log 2-\frac{n}{2} \log 2 \pi \sigma-\frac{1}{2} \sum_{i=1}^{n} \log m_{i}-\frac{1}{2} \sum_{i=1}^{n} B_{i}^{2}+\sum_{i=1}^{n} \log \Phi\left(\lambda B_{i}\right)
$$

em que $B_{i}=\frac{y_{i}-\eta\left(\boldsymbol{\beta}, \boldsymbol{x}_{\boldsymbol{i}}\right)+\sqrt{\frac{2}{\pi}} \Delta m_{i}^{1 / 2}}{m_{i}^{1 / 2} \sigma}$ 


\subsubsection{Erros com distribuição skew t}

O modelo de regressão não linear baseado na distribuição skew t (ST) é definido por

$$
Y_{i}=\eta\left(\boldsymbol{\beta}, x_{i}\right)+\epsilon_{i}, i=1, \ldots, n,
$$

em que $Y_{i}$ é a variável resposta, $\eta(\cdot)$ é uma função injetora, contínua e duas vezes diferenciável com respeito ao vetor de parâmetros $\boldsymbol{\beta}=\left(\beta_{1}, \ldots, \beta_{p}\right)^{T}$, $x_{i}$ é um vetor de variáveis explicativas e erros aleatórios $\epsilon_{i} \sim S N\left(-\sqrt{\frac{2}{\pi}} k_{1} \Delta m_{i}^{1 / 2}, m_{i} \sigma^{2}, \lambda, H\right)$, em que $m_{i}=m\left(\rho, x_{i}\right)$ é uma função conhecida, diferenciável, contínua e positiva e $\boldsymbol{x}_{i}=$ $\left(x_{i 1}, \ldots, x_{i q}\right)^{T}$ são valores da variável explicativa e $\rho$ um parâmetro desconhecido.

Para o modelo (5.4), temos que

$$
E\left[Y_{i}\right]=\eta\left(\boldsymbol{\beta}, x_{i}\right), \operatorname{Var}\left[Y_{i}\right]=m_{i} \sigma^{2}\left(k_{2}-b^{2} \delta^{2}\right),
$$

em que $b=-\sqrt{\frac{2}{\pi}} k_{1}$ e $Y_{i} \sim S T\left(\eta\left(\beta, x_{i}\right)+b \Delta m_{i}^{1 / 2}, m_{i} \sigma^{2}, \lambda\right)$, para $i=1, \ldots, n$.

Segue que a função log-verossimilhança para $\boldsymbol{\theta}=\left(\boldsymbol{\beta}^{T}, \sigma^{2}, \lambda, \nu^{T}\right)^{T}$, dada pela amostra observada $\boldsymbol{y}=\left(y_{1}, \ldots, y_{n}\right)^{T}$ é dada por

$l(\boldsymbol{\theta})=n \log \left(\frac{\nu+1}{2}\right)-n \log \frac{\Gamma(\nu)}{2}-\frac{n}{2} \log \pi \nu \sigma^{2}-\frac{1}{2} \sum_{i=1}^{n} \log m_{i}-\frac{\nu+1}{2} \sum_{i=1}^{n} \log \left(1+d_{i}\right) \sum_{i=1}^{n} \log K_{i}$,

em que $K_{i}=T\left(\sqrt{\frac{\nu+1}{d_{i}+\nu}} A_{i} ; \nu+1\right)$ e $d_{i}=\left(y_{i}-\eta\left(\beta, x_{i}\right)+b \Delta m_{i}^{1 / 2}\right)^{2} /\left(m_{i} \sigma^{2}\right)$ e $A_{i}=d_{i}^{1 / 2} \lambda$. 


\subsection{Critérios de seleção de modelos}

Um modelo é a representação simplificada de algum problema ou situação da vida real destinado a ilustrar certos aspectos do problema sem se ater a todos os detalhes. Não raro, mais de um modelo pode descrever um mesmo fenômeno, haja vista que cada pesquisador tem a liberdade de modelar o fenômeno seguindo a metodologia que julgar mais adequada.

Existe uma variedade de metodologia para comparar modelos concorrentes para um conjunto de dados e selecionar aquele que melhor se ajusta aos dados. Um dos mais usados nas aplicações é a densidade preditiva condicional ordenada (CPO). Para discussões detalhadas da estatística $C P O_{i}$ e suas aplicações para seleção de modelos, ver Gelfand et al. (1992).

Seja $\mathbf{D}$ os dados completos e $\mathbf{D}^{(-i)}$ os dados com a i-ésima observação excluída. Denotamos a densidade a posteriori de $\boldsymbol{\theta}$ dado $\mathbf{D}^{(-i)}$ por $\pi\left(\boldsymbol{\theta} \mid \mathbf{D}^{(-i)}\right)$, para $i=1, \ldots, n$. Para a i-ésima observação, a $C P O_{i}$ pode ser escrita como

$$
C P O_{i}=\int_{\Theta} g\left(y_{i} \mid \boldsymbol{\theta}\right) \pi\left(\boldsymbol{\theta} \mid \mathbf{D}^{(-i)}\right) d \boldsymbol{\theta}=\left\{\int_{\Theta} \frac{\pi(\boldsymbol{\theta} \mid \mathbf{D})}{g\left(y_{i} \mid \boldsymbol{\theta}\right)} d \boldsymbol{\theta}\right\}^{-1}, i=1, \ldots, n
$$

em que $g\left(y_{i} \mid \boldsymbol{\theta}\right)$ é a função densidade de probabilidade.

A $C P O_{i}$ pode ser interpretada como o peso da densidade marginal do tempo para o evento em $y_{i}$. Deste modo, valores altos de $C P O_{i}$ implicam em melhor ajuste para o modelo. Para os modelos propostos, vistos na seção anterior, uma forma fechada da $C P O_{i}$ não está disponível. Entretanto, uma estimativa de Monte Carlo da $C P O_{i}$ pode ser obtida usando amostras MCMC da distribuição a posteriori $\pi(\boldsymbol{\theta} \mid \mathbf{D})$. Seja $\boldsymbol{\theta}_{1}, \ldots, \boldsymbol{\theta}_{Q}$ uma amostra de tamanho $Q$ de $\pi(\boldsymbol{\theta} \mid \mathbf{D})$ depois do burn-in. Uma aproximação de Monte Carlo da $C P O_{i}$ (Chen et al. (2000)) é dada por 


$$
\widehat{C_{P O}}=\left\{\frac{1}{Q} \sum_{q=1}^{Q} \frac{1}{g\left(y_{i} \mid \boldsymbol{\theta}_{q}\right)}\right\}^{-1} .
$$

O sumário estatístico da $C P O_{i}$ é $B=\sum_{i=1}^{n} \log \left(\widehat{C P O}_{i}\right)$. Do mesmo modo, quanto maior for o valor de $B$, melhor é o ajuste do modelo aos dados.

Outros critérios podem ser usados, como o critério de informação do desvio (DIC), proposto por Spiegelhalter et al. (2002), o critério de informação de Akaike esperado (EAIC), proposto por Brooks (2002) e o critério de informação Bayesiano (ou de Schwarz) esperado (EBIC), proposto por Carlin \& Louis (2001).

O DIC é uma generalização hierárquica do AIC e do BIC. É muito usado na seleção de modelos Bayesianos em que a distribuição a posteriori dos modelos é obtida pela simulação de Monte Carlo via cadeia de Markov (MCMC). A ideia é que modelos com menor DIC devem ser escolhidos frente aos modelos com maior DIC.

A vantagem do DIC em relação aos outros critérios, é que ele é facilmente calculado de amostras geradas por MCMC, enquanto o AIC e o BIC requerem o cálculo da verossimilhança no valor máximo do parâmetro em questão.

O critério de informação Akaike foi desenvolvido por Hirotsugu Akaike, sendo atrelado ao conceito de entropia, de fato oferecendo uma medida relativa da informação perdida quando um modelo é usado para descrever a realidade. Pode ser dito que é para descrever a troca entre vício e a variância na construção do modelo, ou livremente falando, da precisão e complexidade do modelo.

O AIC não é um teste no modelo no sentido de teste de hipóteses, ele fornece a média para comparação entre os modelos - uma ferramenta para modelo de seleção. Dado um conjunto de dados, diversos modelos podem ser classificados de acordo com seu AIC, o modelo que tiver menor AIC, será considerado o melhor. 
O BIC, desenvolvido por Schwarz (1978), é um critério de seleção de modelos entre uma classe de modelos paramétricos com diferentes números de parâmetros. Escolher um modelo para otimizar o BIC, é uma forma de regularização.

Quando os parâmetros de um modelo são estimados usando a estimação de máxima verossimilhança, é possível aumentar a verossimilhança adicionando parâmetros, o qual pode resultar em super estimação. O BIC resolve este problema introduzindo uma penalidade para o número de parâmetros do modelo. Esta penalidade é maior no BIC que no AIC.

Assim, esses critérios são baseados na média a posteriori do desvio, que pode ser aproximado por $\bar{D}=\sum_{q=1}^{Q} D\left(\boldsymbol{\theta}_{q}\right) / Q$, em que $D(\boldsymbol{\theta})=-2 \sum_{i=1}^{n} \log \left[g\left(y_{i} \mid \boldsymbol{\theta}\right)\right]$. O DIC pode ser estimado usando saídas MCMC calculando $\widehat{D I C}=\bar{D}+\widehat{\rho_{D}}=2 \bar{D}-\widehat{D}$, em que $\widehat{\rho_{D}}$ é o número de parâmetros, no qual é definido como $E[D(\boldsymbol{\theta})]-D[E(\boldsymbol{\theta})]$, em que $D[E(\boldsymbol{\theta})]$ é o desvio esperado na média a posteriori e pode ser obtido da seguinte forma

$$
\widehat{D}=D\left(\frac{1}{Q} \sum_{q=1}^{Q} \boldsymbol{\mu}_{q}, \frac{1}{Q} \sum_{q=1}^{Q} \lambda_{q}, \frac{1}{Q} \sum_{q=1}^{Q} \sigma_{q}^{2}, \frac{1}{Q} \sum_{q=1}^{Q} \boldsymbol{\nu}_{q}\right)
$$

Similarmente, os critérios EAIC e EBIC podem ser estimados por

$$
\begin{gathered}
\widehat{E A I C}=\bar{D}+2 \#(\vartheta) \\
\widehat{E B I C}=\bar{D}+\#(\vartheta) \log (n),
\end{gathered}
$$

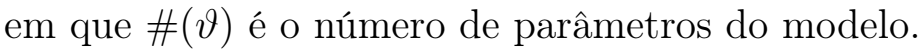

As mesmas interpretações são adotadas no contexto bayesiano para os critérios EAIC e EBIC. 


\subsection{Diagnóstico}

A identificação de observações influentes é importante na análise estatística, e o desenvolvimento de medidas diagnósticas para detectar observações influentes é de interesse para muitos pesquisadores. Observações influentes em um conjunto de dados podem ter um grande impacto na inferência estatística, conclusões e pode indicar falta de adequação do modelo aos dados em questão. Assim, as observações influentes são partes importantes nos dados e requerem cuidadosa examinação.

Uma maneira de avaliar a influência das observações no modelo ajustado é através de medidas de diagnóstico globais, como a deleção de casos. Na análise Bayesiana, a divergência de Kullback-Leibler (K-L), baseada na deleção de casos, é a medida de discrepância entre as distribuições a posteriori com e sem a exclusão do caso particular.

Seja $\mathbf{D}$ os dados completos e $\mathbf{D}^{(-i)}$ os dados com a i-ésima observação excluída. Denotamos a densidade a posteriori de $\boldsymbol{\theta}$ dado $\mathbf{D}^{(-i)}$ por $\pi\left(\boldsymbol{\theta} \mid \mathbf{D}^{(-i)}\right)$, para $i=1, \ldots, n$.

Suponha que $K\left(P ; P_{(-i)}\right)$ denota a divergência K-L entre $\mathrm{P}$ e $P_{(-i)}$, em que $\mathrm{P}$ é a distribuição a posteriori de $\theta$ para os dados completos e $P_{(-i)}$ é a distribuição a posteriori de $P_{(-i)}$ sem o i-ésimo caso. Especificamente,

$$
K\left(P ; P_{(-i)}\right)=\int \pi(\boldsymbol{\theta} \mid \mathbf{D}) \log \left[\frac{\pi(\boldsymbol{\theta} \mid \mathbf{D})}{\pi\left(\boldsymbol{\theta} \mid \mathbf{D}^{(-i)}\right)}\right] d \boldsymbol{\theta} .
$$

A quantidade $K\left(P ; P_{(-i)}\right)$ mede o efeito de omitir o i-ésimo caso dos dados completos na distribuição a posteriori de $\theta$. Como mostrado em Peng \& Dey (1995) e Cho et al. (2009), a calibração de $K\left(P ; P_{(-i)}\right)$ pode ser feita resolvendo a $p_{i}$ da equação $K\left(P ; P_{(-i)}\right)=K\left[B(0.5), B\left(p_{i}\right)\right]=-\log \left[4 p_{i}\left(1-p_{i}\right)\right] / 2$, em que $B(p)$ denota a distribuição Bernoulli com probabilidade de sucesso $p$. Isto implica que descrever resultados usando $\pi(\boldsymbol{\theta} \mid \mathbf{D})$ ao invés de $\pi\left(\boldsymbol{\theta} \mid \mathbf{D}^{(-i)}\right)$ é equivalente a descrever um evento não ob- 
servado com probabilidade $p_{i}$, quando a probabilidade correta é 0.5. Após algumas manipulações algébricas, pode ser mostrado que $\left.p_{i}=\frac{1}{2}\left\{1+\sqrt{1-\exp \left[-2 K\left(P ; P_{(-i)}\right)\right.}\right]\right\}$. Esta equação implica que $0.5 \leq p_{i} \leq 1$. Além disso, se $p_{i}>>0.5$, implica que o i-ésimo caso é influente.

Para os modelos assimétricos heteroscedásticos utilizados, a equação (5.6) pode ser expressa como a média a posteriori

$$
\begin{aligned}
K\left(P ; P_{(-i)}\right) & =\log E_{\theta \mid \mathbf{D}}\left\{\left[g\left(y_{i} \mid \boldsymbol{\theta}\right)\right]^{-1}\right\}+E_{\theta \mid \mathbf{D}}\left\{\left[\log g\left(y_{i} \mid \boldsymbol{\theta}\right)\right]\right\} \\
& =-\log \left(C P O_{i}\right)+E_{\theta \mid \mathbf{D}}\left\{\left[\log g\left(y_{i} \mid \boldsymbol{\theta}\right)\right]\right\}
\end{aligned}
$$

em que $E_{\theta \mid \mathbf{D}}(\cdot)$ denota a esperança com respeito a posteriori $\pi(\theta \mid \mathbf{D})$. Amostras da distribuição a posteriori de $\boldsymbol{\theta}$ para (5.7) podem ser calculadas via o método MCMC. Seja $\theta_{1}, \ldots, \theta_{Q}$ uma amostra de tamanho $Q$ de $\pi(\theta \mid \mathbf{D})$. A estimativa de Monte Carlo de $K\left(P ; P_{(-i)}\right)$ é dada por

$$
K\left(P ; \hat{P}_{(-i)}\right)=-\log \left(\widehat{C P O_{i}}\right)+\frac{1}{Q} \sum_{q=1}^{Q} \log \left[g\left(y_{i} \mid \theta_{q}\right)\right]
$$

sendo $\widehat{\mathrm{CPO}_{i}}$ como descrito em (5.5). 


\section{Capítulo 6}

\section{Aplicação}

Neste capítulo ilustraremos a metodologia descrita anteriormente com um conjunto de dados simulados e em seguida faremos uma aplicação para um conjunto de dados reais. Primeiramente, faremos um estudo de simulação para exemplificar a abordagem proposta e o uso dos critérios de seleção dos modelos citados anteriormente. Isso será feito somente para as distribuições skew normal e skew t considerando apenas o caso heteroscedástico que é o foco deste trabalho. Na aplicação com um conjunto de dados reais, comparamos os modelos simétricos com os assimétricos, considerando tanto o caso homocedástico quanto o heteroscedástico para as distribuições resultante como melhor modelo avaliado pelos critérios de seleção adotados em 5.3. Em seguida é feito um estudo de robustez a fim de verificar suposições feitas para o modelo melhor ajustado aos dados.

\subsection{Estudo com um conjunto de dados simulados}

Para exemplificar a abordagem bayesiana utilizada e o uso dos critérios de seleção de modelos propostos neste trabalho, consideramos um conjunto de dados simulados, em 
que a covariável $x_{i}, i=1, \ldots, 40$ são números aleatórios entre 1 e 5 . A variável resposta foi simulada das distribuições skew normal adotando o modelo de Brody como média da distribuição e função de variância dada por (6.2). Para gerar os parâmetros do modelo assimétrico heteroscedástico utilizamos os valores: $\beta_{1}=35, \beta_{2}=1, \beta_{3}=0,5$, $\sigma^{2}=3, \lambda=-7$ e $\rho=0,8$.

A Tabela 6.1 apresenta os valores dos critérios de seleção considerando os modelos de crescimento Brody, Gompertz, Logístico e Von Bertalanffy.

Tabela 6.1: Sumário dos critérios de seleção com erros normais

\begin{tabular}{ccccc}
\hline & EAIC & EBIC & CPO & DIC \\
\hline Brody & 152,1238 & 162,2571 & $-72,94243$ & 143,7247 \\
Gompertz & 154,2306 & 165,3639 & $-73,96904$ & 145,6048 \\
Logístico & 155,6655 & 165,7987 & $-74,45134$ & 146,9725 \\
Von Bertalanffy & 154,181 & 165,3143 & $-74,0789$ & 145,4754 \\
\hline
\end{tabular}

Como era esperado, verifica-se que o melhor modelo, entre todos utilizados, com erros skew normal é o de Brody, pois tem menor valor para o EAIC, EBIC e DIC, e maior valor para o critério CPO.

O sumário a posteriori do modelo escolhido é mostrado a seguir.

Tabela 6.2: Sumário do modelo para os dados simulados

\begin{tabular}{ccccccccc}
\hline & Média & Desvio Padrão & $2,5 \%$ & $25 \%$ & $50 \%$ & $75 \%$ & $97,5 \%$ & Rhat \\
\hline$\beta_{1}$ & 33,72 & 1,58 & 31,20 & 32,61 & 33,52 & 34,67 & 37,13 & 1,006 \\
$\beta_{2}$ & 1,15 & 0,10 & 0,94 & 1,08 & 1,15 & 1,21 & 1,33 & 1,002 \\
$\beta_{3}$ & 0,61 & 0,09 & 0,42 & 0,55 & 0,61 & 0,67 & 0,78 & 1,004 \\
$\lambda$ & $-8,73$ & 6,55 & $-25,57$ & $-12,32$ & $-6,85$ & $-3,71$ & $-0,06$ & 1,009 \\
$\sigma^{2}$ & 1,90 & 1,31 & 0,44 & 0,99 & 1,59 & 2,46 & 4,89 & 1,002 \\
$\rho$ & 1,20 & 0,61 & 0,00 & 0,77 & 1,17 & 1,63 & 2,39 & 1,003 \\
\hline
\end{tabular}

Com a tabela acima, verifica-se que os valores utilizados para gerar os dados estão próximos dos valores estimados, e portanto, dentro do intervalo de confiança. 


\subsection{Aplicação para um conjunto de dados}

Os dados utilizados para testar a metodologia proposta foram coletados em florestas plantadas de clones de Eucalyptus sp. da Votorantim Celulose e Papel (VCP), na região de Itapetininga, sul do estado de São Paulo, com coordenadas geográficas dadas por latitude $23^{\circ} 56^{\prime} S$, longitude $42^{\circ} 211^{\prime} W$ e altitude média de $655 \mathrm{~m}$. Os solos são predominantemente Latossolo Vermelho distrófico, descrevendo uma situação favorável à cultura de Eucalyptus sp.

A Figura 6.1 mostra o histograma dos dados e um gráfico normal Q-Q plot, em que temos indícios que os dados são assimétricos.

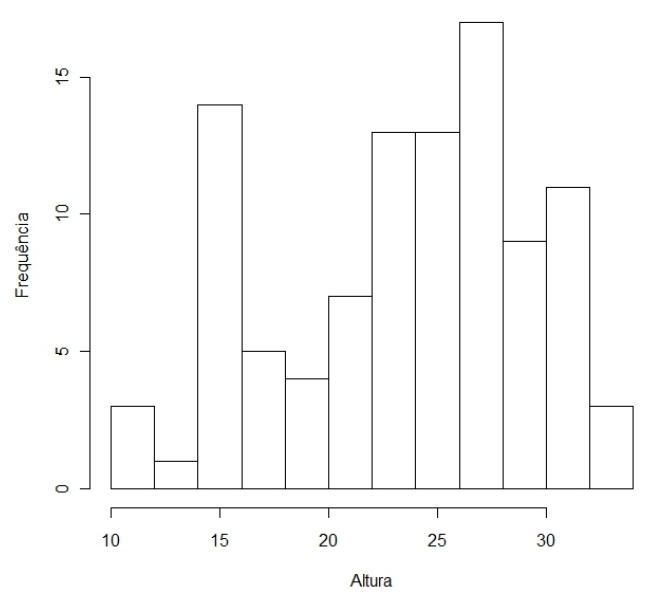

(a) Histograma dos dados

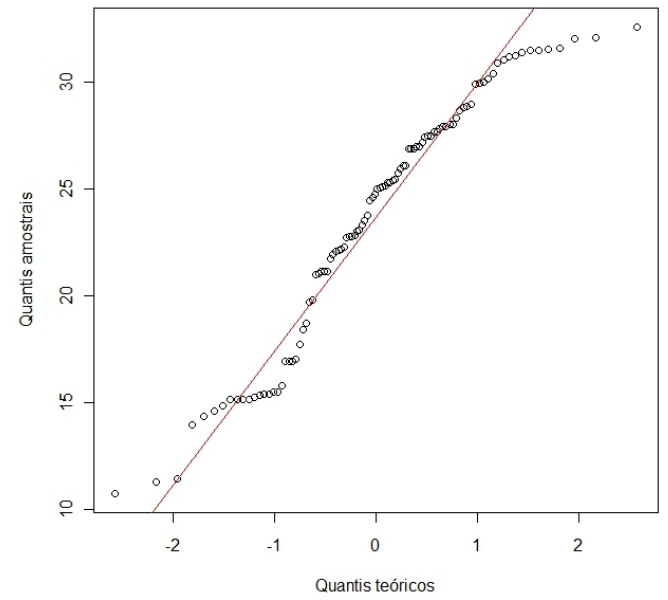

(b) Normal Q-Q Plot

Figura 6.1: Histograma e normal Q-Q plot dos dados arbóreos.

Para efeito de comparação, utilizamos as distribuições normal e t-Student em contraste com sua forma assimétrica a fim de verificarmos que os modelos de regressão não linear com erros assimétricos são os que melhor se ajustam a esses dados.

As estimativas bayesianas foram obtidas usando as seguintes prioris independentes. Apesar dos parâmetros não poderem assumir valores negativos, foi adotado uma priori Normal com uma variância grande para obtermos um caráter informativo vago a estes 
parâmetros.

$$
\beta_{j} \sim N\left(0,10^{3}\right), j=1,2,3
$$

para os parâmetros dos modelos de crescimento,

$$
\sigma^{2} \sim I G(1,0.1)
$$

para o parâmetro de escala,

$$
\lambda \sim N\left(0,10^{3}\right)
$$

para o parâmetro de assimetria, e

$$
\nu \sim \operatorname{Exp}(0.5) I_{\{\nu>2\}}
$$

para o parâmetro de curtose.

\subsubsection{Caso homocedástico}

Para cada distribuição considerada (normal, t-Student, skew normal e skew-t), apresentamos uma tabela com os valores dos critérios de seleção utilizados para cada modelo de crescimento (Brody, Gompertz, Logístico e Von Bertalanffy). Em seguida, com a escolha do melhor modelo, uma tabela com a média, o desvio padrão, os percentis e o $\hat{R}$ dos parâmetros é exibida.

- Distribuição Normal

Pela tabela abaixo, há evidências de que o melhor modelo, entre todos utilizados, com erros distribuídos normalmente é o de Brody, pois tem menor valor para o 
EAIC, EBIC e DIC, e maior valor para o critério CPO.

Tabela 6.3: Sumário dos critérios de seleção com erros normais

\begin{tabular}{ccccc}
\hline & EAIC & EBIC & CPO & DIC \\
\hline Brody & 399,1988 & 409,6195 & $-197,8583$ & 394,4037 \\
Gompertz & 400,6215 & 411,0421 & $-198,5646$ & 396,5385 \\
Logístico & 402,4405 & 412,8612 & $-199,4883$ & 398,3902 \\
Von Bertalanffy & 400,0464 & 410,4671 & $-198,2693$ & 395,7322 \\
\hline
\end{tabular}

A seguir apresentamos as estimativas a posteriori do modelo escolhido.

Tabela 6.4: Sumário do modelo de Brody com erros normais

\begin{tabular}{ccccccccc}
\hline & Média & Desvio Padrão & $2,5 \%$ & $25 \%$ & $50 \%$ & $75 \%$ & $97,5 \%$ & $\hat{R}$ \\
\hline$\beta_{1}$ & 35,44 & 2,04 & 32,30 & 34,00 & 35,16 & 36,55 & 39,86 & 1,001 \\
$\beta_{2}$ & 1,20 & 0,11 & 1,02 & 1,12 & 1,19 & 1,26 & 1,45 & 1,002 \\
$\beta_{3}$ & 0,33 & 0,05 & 0,24 & 0,29 & 0,33 & 0,37 & 0,44 & 1,001 \\
$\sigma^{2}$ & 2,91 & 0,44 & 2,18 & 2,59 & 2,88 & 3,17 & 3,83 & 1,001 \\
\hline
\end{tabular}

- t-Student

A tabela 6.5 apresenta os resultados dos critérios de seleção para cada modelo de crescimento considerando a distribuição t-Student para os erros.

Tabela 6.5: Sumário dos critérios de seleção com erros t-Student

\begin{tabular}{ccccc}
\hline & EAIC & EBIC & CPO & DIC \\
\hline Brody & 398,36 & 411,3859 & $-196,6566$ & 391,8776 \\
Gompertz & 401,4683 & 414,4941 & $-198,3929$ & 395,8071 \\
Logístico & 404,4376 & 417,4634 & $-199,8813$ & 398,959 \\
Von Bertalanffy & 400,3947 & 413,4206 & $-197,7714$ & 394,4498 \\
\hline
\end{tabular}

Há evidências de que o melhor modelo, entre todos utilizados, com erros t-Student é o de Brody, pois tem menor valor para o EAIC, EBIC e DIC, e maior valor para o critério CPO.

As estimativas a posteriori do modelo escolhido são apresentadas na tabela 6.6. 
Tabela 6.6: Sumário do modelo de Brody com erros t-Student

\begin{tabular}{ccccccccc}
\hline & Média & Desvio Padrão & $2,5 \%$ & $25 \%$ & $50 \%$ & $75 \%$ & $97,5 \%$ & $\hat{R}$ \\
\hline$\beta_{1}$ & 35,99 & 1,85 & 33,01 & 34,69 & 35,74 & 37,08 & 40,26 & 1,000 \\
$\beta_{2}$ & 1,21 & 0,10 & 1,04 & 1,13 & 1,20 & 1,27 & 1,43 & 1,000 \\
$\beta_{3}$ & 0,33 & $0 ., 05$ & 0,24 & 0,30 & 0,33 & 0,36 & 0,43 & 1,000 \\
$\sigma^{2}$ & 1,53 & 0,39 & 0,91 & 1,24 & 1,47 & 1,75 & 2,38 & 1,002 \\
$\nu$ & 3,73 & 1,41 & 2,09 & 2,73 & 3,39 & 4,34 & 7,56 & 1,000 \\
\hline
\end{tabular}

- Distribuição Skew Normal

A Tabela 6.7 apresenta os resultados dos critérios de seleção para cada modelo de crescimento considerando a distribuição skew normal para os erros.

Tabela 6.7: Sumário dos critérios de seleção com erros skew normal

\begin{tabular}{ccccc}
\hline & EAIC & EBIC & CPO & DIC \\
\hline Brody & 377,5026 & 390,5284 & $-186,0595$ & 371,1544 \\
Gompertz & 379,2268 & 392,2526 & $-186,9741$ & 373,7046 \\
Logístico & 381,9564 & 394,9822 & $-188,5308$ & 376,4147 \\
Von Bertalanffy & 378,3178 & 391,3436 & $-186,6195$ & 372,4395 \\
\hline
\end{tabular}

Há indícios de que o melhor modelo, entre todos utilizados, com erros skew normal é o de Brody, pois tem menor valor para o EAIC, EBIC e DIC, e maior valor para o critério CPO.

A seguir apresentamos as estimativas a posteriori do modelo escolhido.

Tabela 6.8: Sumário do modelo de Brody com erros skew normal

\begin{tabular}{ccccccccc}
\hline & Média & Desvio Padrão & $2,5 \%$ & $25 \%$ & $50 \%$ & $75 \%$ & $97,5 \%$ & $\hat{R}$ \\
\hline$\beta_{1}$ & 37,13 & 1,69 & 34,41 & 35,97 & 36,90 & 38,06 & 41,17 & 1,000 \\
$\beta_{2}$ & 1,14 & 0,07 & 1,02 & 1,10 & 1,14 & 1,18 & 1,28 & 1,000 \\
$\beta_{3}$ & 0,29 & 0,04 & 0,22 & 0,27 & 0,29 & 0,32 & 0,36 & 1,000 \\
$\lambda$ & $-7,65$ & 3,48 & $-16,20$ & $-9,49$ & $-6,77$ & $-5,07$ & $-3,41$ & 1,002 \\
$\sigma^{2}$ & 7,44 & 1,26 & 5,35 & 6,49 & 7,33 & 8,24 & 10,23 & 1,000 \\
\hline
\end{tabular}

- Distribuição Skew t 
A tabela 6.9 apresenta os resultados dos critérios de seleção para cada modelo de crescimento considerando a distribuição skew t para os erros.

Tabela 6.9: Sumário dos critérios de seleção com erros skew-t

\begin{tabular}{ccccc}
\hline & EAIC & EBIC & CPO & DIC \\
\hline Brody & 381,6053 & 397,2363 & $-187,1925$ & 374,6481 \\
Gompertz & 384,3285 & 399,9595 & $-188,5334$ & 377,7788 \\
Logístico & 387,733 & 403,364 & $-190,2386$ & 381,1701 \\
Von Bertalanffy & 383,4441 & 399,0751 & $-188,11$ & 376,9093 \\
\hline
\end{tabular}

Há evidências de que o melhor modelo, entre todos utilizados, com erros skew t é o de Brody, pois tem menor valor para o EAIC, EBIC e DIC, e maior valor para o critério CPO.

As estimativas a posteriori do modelo escolhido são apresentadas na tabela 6.10.

Tabela 6.10: Sumário do modelo de Brody com erros skew-t

\begin{tabular}{ccccccccc}
\hline & Média & Desvio Padrão & $2,5 \%$ & $25 \%$ & $50 \%$ & $75 \%$ & $97,5 \%$ & $\hat{R}$ \\
\hline$\beta_{1}$ & 37,02 & 1,75 & 34,20 & 35,78 & 36,80 & 38,02 & 41,21 & 1.004 \\
$\beta_{2}$ & 1,14 & 0,07 & 1,02 & 1,10 & 1,14 & 1,19 & 1,29 & 1,003 \\
$\beta_{3}$ & 0,29 & 0,04 & 0,22 & 0,27 & 0,29 & 0,32 & 0,37 & 1,004 \\
$\lambda$ & $-3,28$ & 1,14 & $-5,71$ & $-4,02$ & $-3,18$ & $-2,47$ & $-1,31$ & 1,000 \\
$\sigma^{2}$ & 3,73 & 1,14 & 1,84 & 2,93 & 3,63 & 4,38 & 6,22 & 1,002 \\
$\nu$ & 4,87 & 2,20 & 2,29 & 3,37 & 4,35 & 5,71 & 10,90 & 1,002 \\
\hline
\end{tabular}

Para o caso homocedástico o modelo que melhor se ajustou aos dados foi o modelo de crescimento de Brody com os erros skew normal. Com isso temos fortes evidências de que os modelos assimétricos são mais adequados do que os simétricos nesse caso.

Para efeito de ilustração, a Figura 6.2 apresenta os histogramas dos parâmetros do modelo, $\beta_{1}, \beta_{2}, \beta_{3}, \lambda$ e $\sigma^{2}$. 

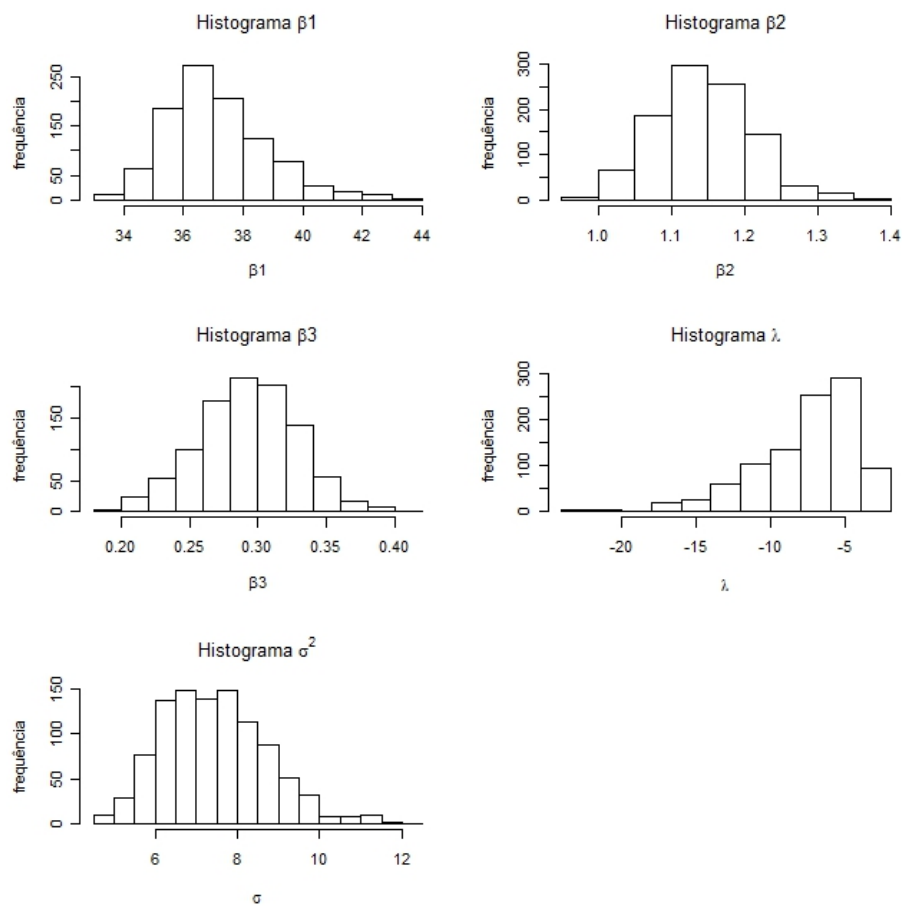

Figura 6.2: Histogramas dos parâmetros do modelo de Brody com erros skew normal.

As séries são mostradas na Figura 6.3.
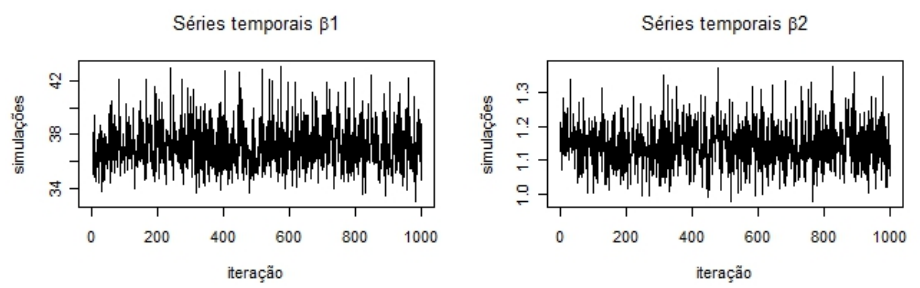

Séries temporais $\beta 3$

Séries temporais $\lambda$
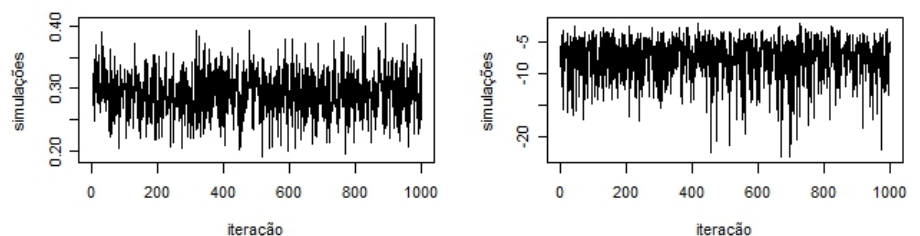

Séries temporais $\sigma^{2}$

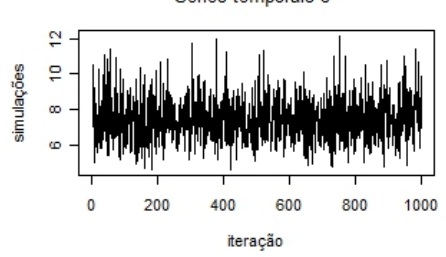

Figura 6.3: Série dos parâmetros do modelo de Brody com erros skew normal. 


\subsubsection{Caso heteroscedástico}

Uma vez que no caso homocedástico o modelo que se melhor ajustou aos dados foi o de Brody, consideramos agora para o caso heteroscedástico somente este modelo com erros seguindo as distribuições skew normal e skew t.

Com a finalidade de compreendermos a variação dos dados, adotamos duas funções para o parâmetro de dispersão dos dados. Essas funções são dadas por

$$
m_{i}=\exp \left(\rho x_{i}\right)
$$

e

$$
m_{i}=x_{i}^{\rho}, \text { para } i=1, \ldots, n
$$

- Distribuição Skew Normal

Primeiro, apresentaremos os sumários do modelo de Brody considerando os erros skew normal e a função $m_{i}=\exp \left(\rho x_{i}\right)$.

Tabela 6.11: Sumário do modelo de Brody com erros skew normal e função de variância $m_{i}=\exp \left(\rho x_{i}\right)$

\begin{tabular}{ccccccccc}
\hline & Média & Desvio Padrão & $2,5 \%$ & $25 \%$ & $50 \%$ & $75 \%$ & $97,5 \%$ & $\hat{R}$ \\
\hline$\beta_{1}$ & 35,56 & 1,63 & 32,77 & 34,47 & 35,43 & 36,44 & 39,17 & 1,003 \\
$\beta_{2}$ & 1,16 & 0,07 & 1,04 & 1,11 & 1,15 & 1,19 & 1,30 & 1,001 \\
$\beta_{3}$ & 0,32 & 0,04 & 0,24 & 0,29 & 0,32 & 0,34 & 0,39 & 1,002 \\
$\lambda$ & $-8,42$ & 3,59 & $-16,75$ & $-10,41$ & $-7,29$ & $-5,97$ & $-3,63$ & 1,000 \\
$\sigma^{2}$ & 3,19 & 1,28 & 1,44 & 2,23 & 2,90 & 3,85 & 6,15 & 1,001 \\
$\rho$ & 0,21 & 0,09 & 0,06 & 0,15 & 0,21 & 0,27 & 0,38 & 1,001 \\
\hline
\end{tabular}

A seguir, apresentamos o sumário do modelo de Brody com erros skew normal, porém com função de variância $m_{i}=x_{i}^{\rho}$. 
Tabela 6.12: Sumário do modelo de Brody com erros skew normal e função de variância $m_{i}=x_{i}^{\rho}$

\begin{tabular}{ccccccccc}
\hline & Média & Desvio Padrão & $2,5 \%$ & $25 \%$ & $50 \%$ & $75 \%$ & $97,5 \%$ & $\hat{R}$ \\
\hline$\beta_{1}$ & 35,92 & 1,44 & 33,41 & 34,91 & 35,87 & 36,81 & 38,79 & 1,001 \\
$\beta_{2}$ & 1,14 & 0,05 & 1,04 & 1,10 & 1,13 & 1,17 & 1,25 & 1,001 \\
$\beta_{3}$ & 0,31 & 0,03 & 0,25 & 0,29 & 0,31 & 0,33 & 0,37 & 1,001 \\
$\lambda$ & $-8,12$ & 2,61 & $-14,28$ & $-9,64$ & $-7,97$ & $-6,25$ & $-3,64$ & 1,008 \\
$\sigma^{2}$ & 2,62 & 1,25 & 1,03 & 1,76 & 2,33 & 3,21 & 5,75 & 1,001 \\
$\rho$ & 0,81 & 0,31 & 0,20 & 0,60 & 0,81 & 1,02 & 1,45 & 1,000 \\
\hline
\end{tabular}

A Tabela 6.13 apresenta os resultados dos critérios de seleção para cada modelo de crescimento considerando a distribuição skew normal para os erros e as duas funções de variância dadas em 6.1 e 6.2.

Tabela 6.13: Sumário dos critérios de seleção para os modelos heteroscedásticos com erros skew normal

\begin{tabular}{ccccc}
\hline & EAIC & EBIC & CPO & DIC \\
\hline$m_{i}=\exp \left(\rho x_{i}\right)$ & 376,9823 & 392,6133 & $-185,2245$ & 368,1654 \\
$m_{i}=x_{i}^{\rho}$ & 376,9550 & 392,586 & $-185,0801$ & 367,3754 \\
\hline
\end{tabular}

Considerando o modelo de Brody com os erros seguindo a distribuição skew normal, pelo critério de seleção, o melhor modelo é dado pela função de variância $m_{i}=x_{i}^{\rho}$.

- Distribuição Skew t

O sumário do modelo de Brody considerando erros skew t e função de variância $m_{i}=\exp \left(\rho x_{i}\right)$ são apresentados na tabela abaixo. 
Tabela 6.14: Sumário do modelo de Brody com erros skew t e função de variância $m_{i}=\exp \left(\rho x_{i}\right)$

\begin{tabular}{ccccccccc}
\hline & Média & Desvio Padrão & $2,5 \%$ & $25 \%$ & $50 \%$ & $75 \%$ & $97,5 \%$ & $\hat{R}$ \\
\hline$\beta_{1}$ & 37,56 & 1,72 & 34,73 & 36,33 & 37,35 & 38,55 & 41,50 & 1,000 \\
$\beta_{2}$ & 1,14 & 0,06 & 1,03 & 1,10 & 1,14 & 1,18 & 1,28 & 1,000 \\
$\beta_{3}$ & 0,30 & 0,04 & 0,23 & 0,27 & 0,30 & 0,32 & 0,37 & 1,000 \\
$\lambda$ & $-4,16$ & 1,35 & $-7,33$ & $-4,98$ & $-4,06$ & $-3,17$ & $-1,95$ & 1,001 \\
$\sigma^{2}$ & 2,68 & 1,51 & 0,93 & 1,68 & 2,32 & 3,23 & 6,80 & 1,000 \\
$\nu$ & 10,16 & 5,09 & 3,33 & 7,00 & 8,70 & 12,14 & 24,61 & 1,000 \\
$\rho$ & 0,17 & 0,11 & $-0,05$ & 0,10 & 0,17 & 0,25 & 0,37 & 1,010 \\
\hline
\end{tabular}

A seguir na Tabela 6.15, o sumário das estimativas a posteriori considerando a função de variância $m_{i}=x_{i}^{\rho}$.

Tabela 6.15: Sumário do modelo de Brody com erros skew t e função de variância $m_{i}=x_{i}^{\rho}$

\begin{tabular}{ccccccccc}
\hline & Média & Desvio Padrão & $2,5 \%$ & $25 \%$ & $50 \%$ & $75 \%$ & $97,5 \%$ & $\hat{R}$ \\
\hline$\beta_{1}$ & 37,33 & 1,58 & 34,39 & 36,20 & 37,24 & 38,33 & 40,77 & 1,002 \\
$\beta_{2}$ & 1,14 & 0,07 & 1,03 & 1,09 & 1,13 & 1,18 & 1,30 & 1,001 \\
$\beta_{3}$ & 0,29 & 0,04 & 0,23 & 0,27 & 0,29 & 0,32 & 0,37 & 1,001 \\
$\lambda$ & $-3,76$ & 1,08 & $-6,11$ & $-4,45$ & $-3,73$ & $-2,99$ & $-1,92$ & 1,00 \\
$\sigma^{2}$ & 3,78 & 1,50 & 1,17 & 2,67 & 3,84 & 4,78 & 6,79 & 1,000 \\
$\nu$ & 9,12 & 5,29 & 3,22 & 5,70 & 7,99 & 10,95 & 24,53 & 1,001 \\
$\rho$ & 0,20 & 0,30 & 0,00 & 0,00 & 0,03 & 0,34 & 0,97 & 1,001 \\
\hline
\end{tabular}

A Tabela 6.16 apresenta os resultados dos critérios de seleção para cada modelo de crescimento considerando a distribuição skew-t para os erros e as duas funções de variância dadas em 6.1 e 6.2 .

Tabela 6.16: Sumário dos critérios de seleção para os modelos heteroscedásticos com erros skew-t

\begin{tabular}{ccccc}
\hline & EAIC & EBIC & CPO & DIC \\
\hline$m_{i}=\exp \left(\rho x_{i}\right)$ & 402,7571 & 420,9933 & $-200,6277$ & 390,8444 \\
$m_{i}=x_{i}^{\rho}$ & 388,7589 & 406,9951 & $-190,9223$ & 381,1337 \\
\hline
\end{tabular}

Há evidências de que o melhor modelo, considerando o modelo de Brody assimétrico 
e heteroscedástico, é o modelo com erros skew normal e função de variância dada pela equação 6.2 .

Para efeito de ilustração, a Figura 6.4 apresenta os histogramas dos parâmetros $\beta_{1}$, $\beta_{2}, \beta_{3}, \lambda, \sigma^{2}$ e $\rho$ do modelo heteroscedástico escolhido.
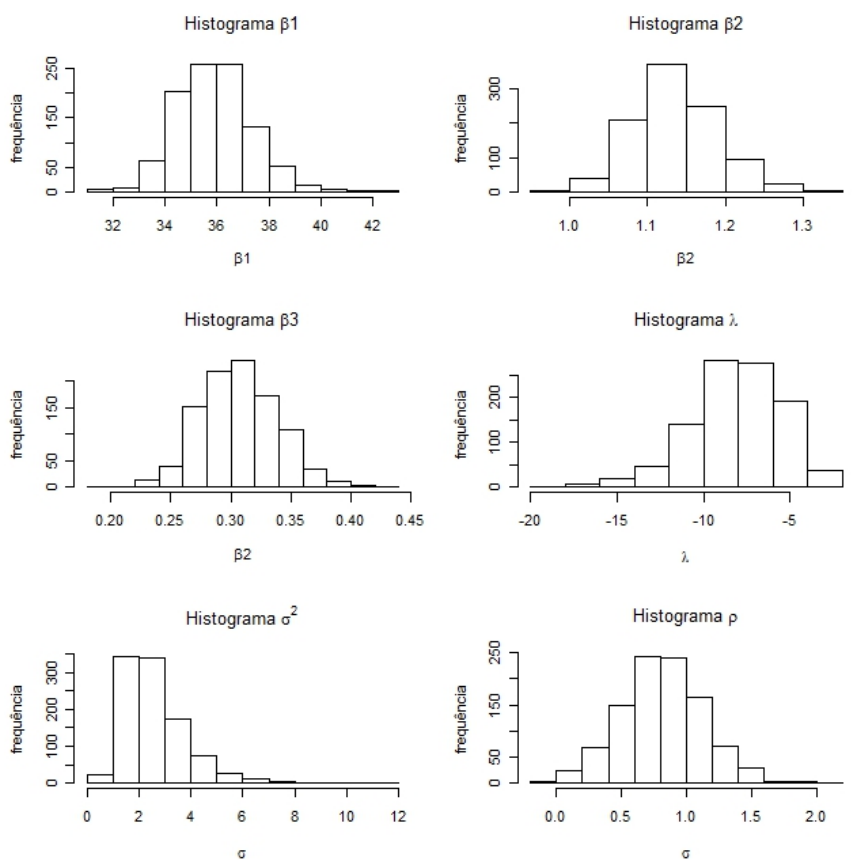

Figura 6.4: Histogramas dos parâmetros do modelo de Brody heteroscedástico com erros skew normal.

As séries são mostradas na Figura 6.5. 

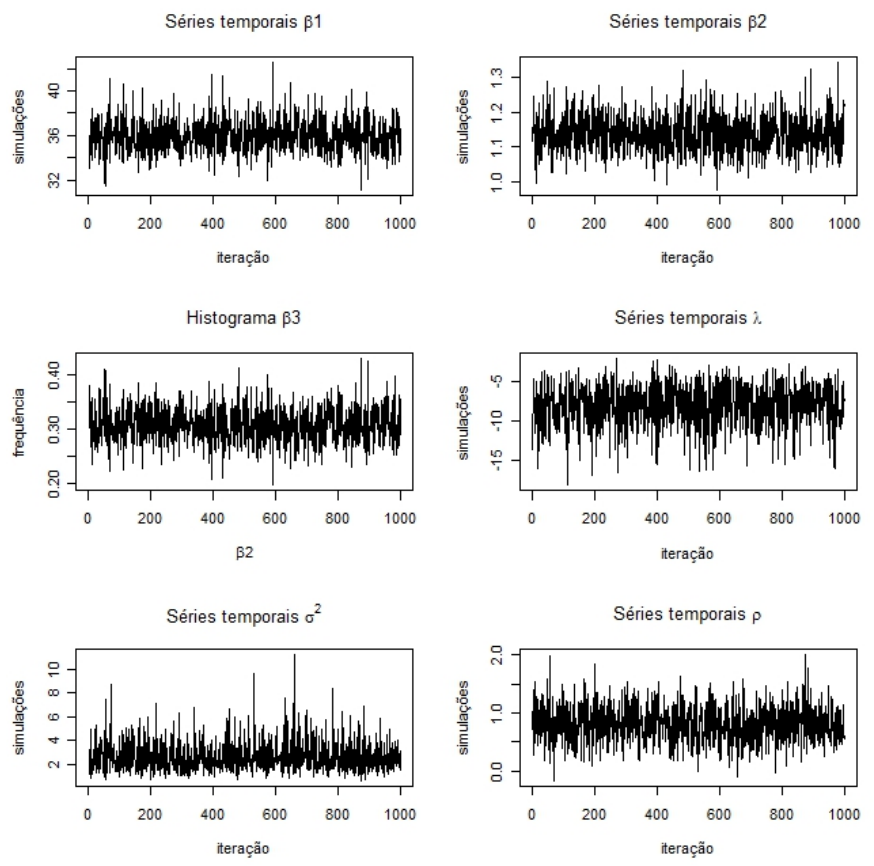

Figura 6.5: Série dos parâmetros do modelo de Brody heteroscedástico com erros skew normal.

Finalmente, a Figura 6.6 mostra o gráfico do ajuste do modelo de Brody com erros skew normal, que resultou no melhor ajuste para os dados utilizados.

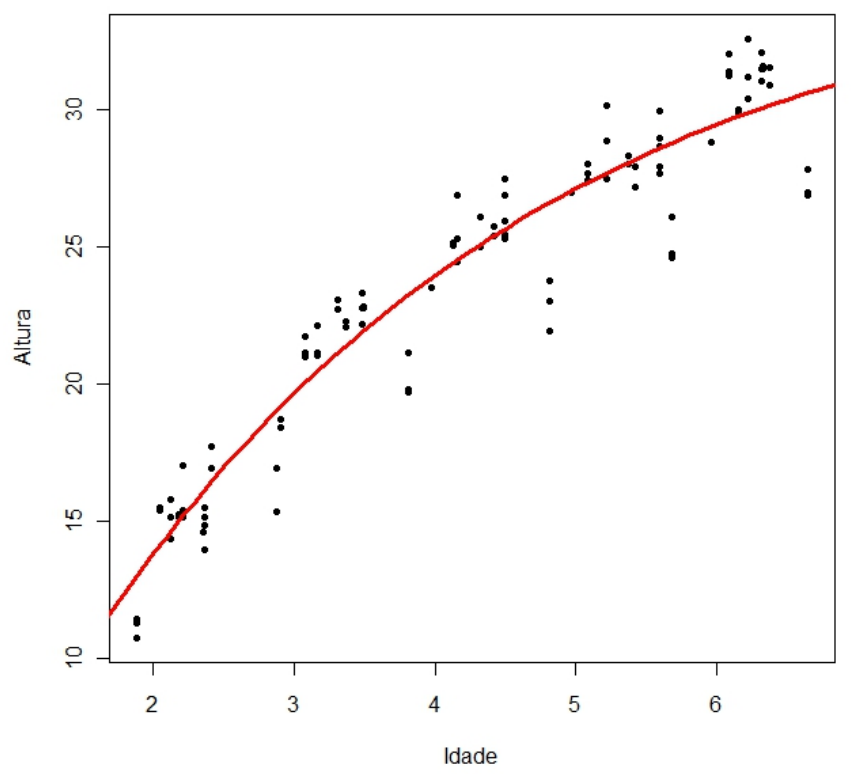

Figura 6.6: Modelo ajustado para os dados arbóreos. 


\subsubsection{Estudo de Robustez}

Com o intuito de verificarmos a influência de uma observação em um modelo ajustado, fizemos um estudo de robustez para os dados utilizados anteriormente, em que $y_{i}$ representa a altura da árvore e $x_{i}$ a idade, para $i=1, \ldots, 100$.

Estudamos a influência de uma mudança em $\Delta_{y}$ unidades em uma observação sob a distribuição a posteriori dos efeitos fixos. Isto é, substituímos o ponto $y_{i}$ pelo valor contaminado $y_{i}=y_{i}+\Delta_{y}$, estimamos o modelo novamente e calculamos a média a posteriori, o desvio padrão, os percentis e o $\hat{R}$. Consideramos a $57^{\circ}$ observação, sendo que o $\Delta$ utilizado foi de 6 unidades. Vale ressaltar que essa discrepância para dados de crescimento pode ser decorrente de um erro de medição.

Como medida de influência de uma observação, consideramos a divergência de Kullback-Leibler, apresentada na seção 5.4.

Uma vez que o modelo de Brody com erros skew normal e função de variância $m_{i}=x_{i}^{\rho}$ foi o modelo escolhido pelos critérios de seleção, ajustamos o mesmo modelo para os dados com a pertubação citada anteriormente.

A Figura 6.7 ilustra os dados com a pertubação na observação 57.

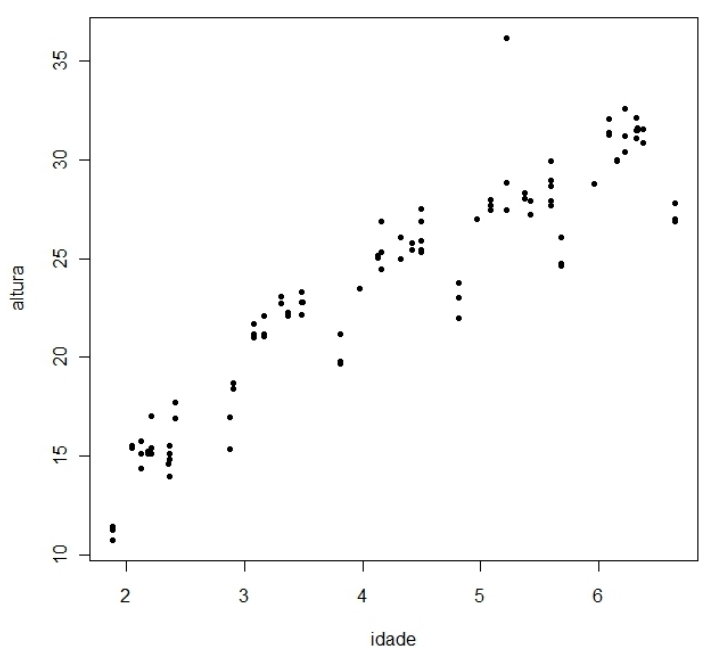

Figura 6.7: Dados com a pertubação de uma observação. 
Os valores dos critérios de seleção para este novo modelo ajustado são dados na tabela abaixo.

Tabela 6.17: Sumário dos critérios de seleção para o modelo com os dados perturbados

\begin{tabular}{cccc}
\hline EAIC & EBIC & CPO & DIC \\
\hline 416.8522 & 432.4832 & -208.4704 & 404.2512 \\
\hline
\end{tabular}

O modelo com a perturbação dos dados se mostra inferior ao modelo ajustado anteriormente quando consideramos os valores dos critérios de seleção.

Na Figura 6.8 ilustramos o gráfico de $K\left(P, P_{(-i)}\right)$ para o modelo proposto, e podemos notar que K-L identifica os casos influentes apresentando valores maiores de $K\left(P, P_{(-i)}\right)$ para o caso perturbado se comparado com as outras observações. Para efeito de comparação, apresentamos primeiro o gráfico de $K\left(P, P_{(-i)}\right)$ para os dados sem a perturbação.
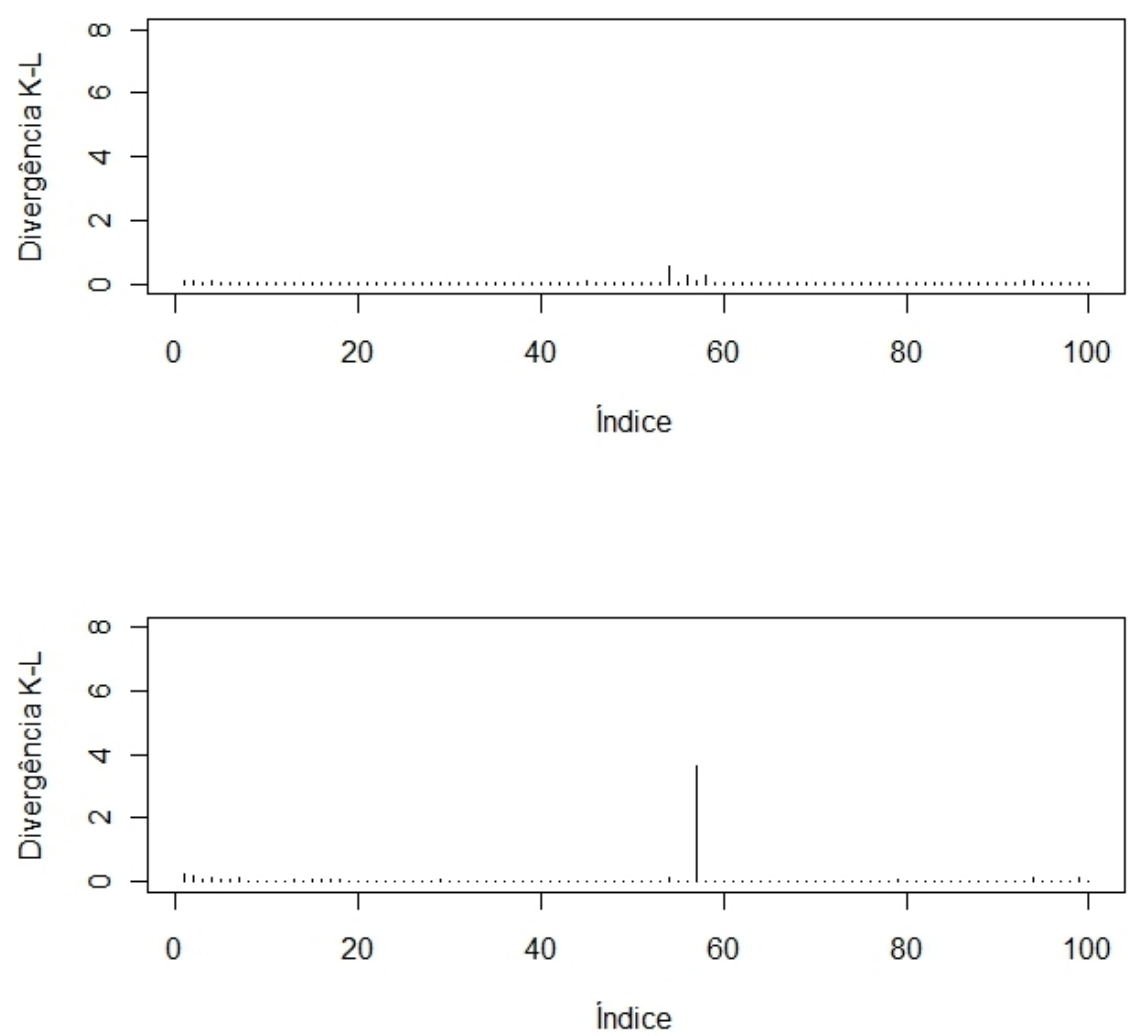

Figura 6.8: Divergência de Kullback-Leibler. 
Podemos verificar que sem a pertubação dos dados utilizados, as observações parecem não ser influentes, pois obtivemos valores pequenos para $K\left(P, P_{(-i)}\right)$. Entretanto após a pertubação, obtivemos uma medida de $K\left(P, P_{(-i)}\right)$ grande para a observação perturbada.

Para verificarmos se essa observação é influente, estimamos a calibração da medida de divergência K-L. A figura abaixo apresenta o gráfico da calibração. Nesta ilustração, confirmamos que a observação perturbada é influente.
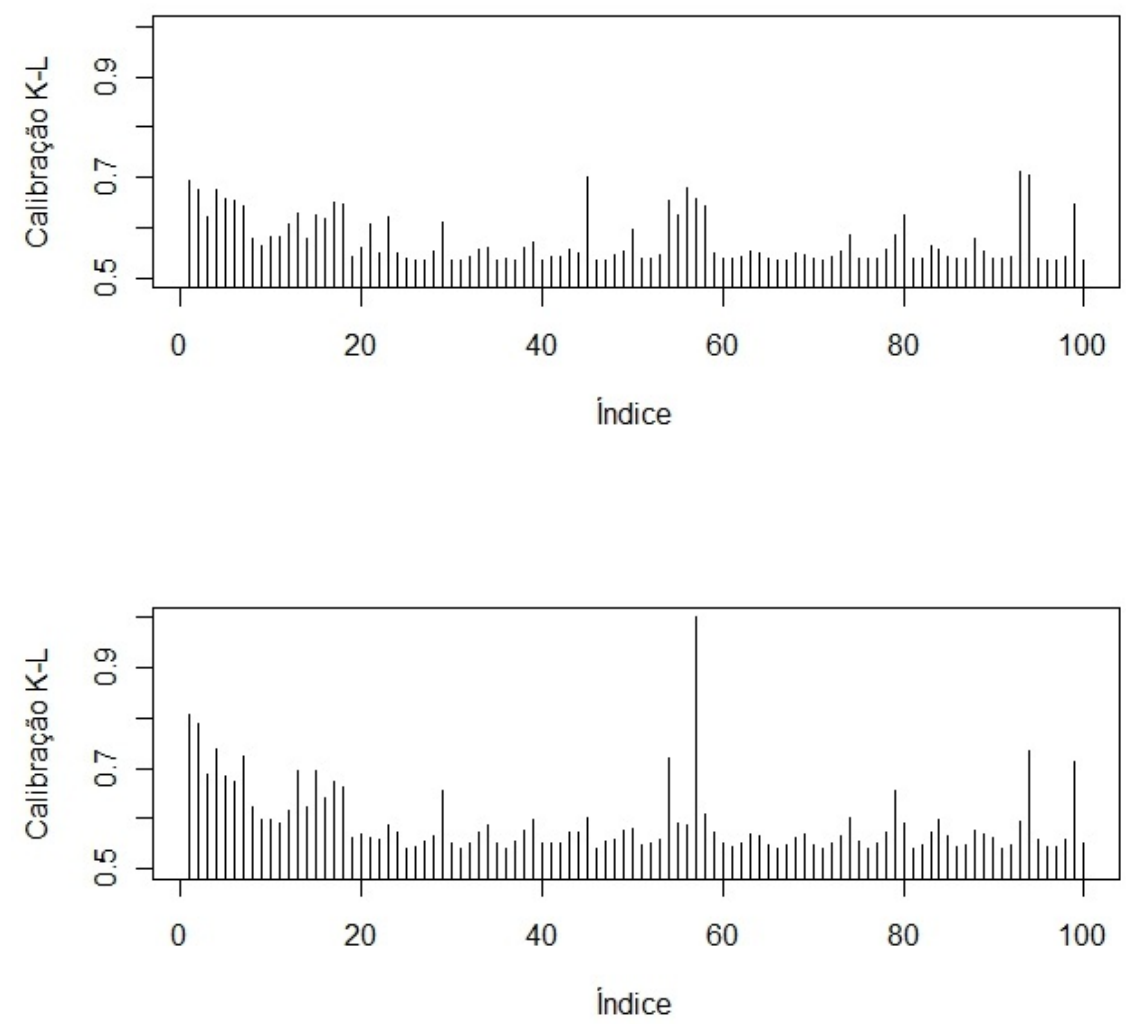

Figura 6.9: Calibração da divergência K-L.

Essa estimativa é apresentada na Tabela 6.18 conjuntamente com a respectiva estimativa da divergência K-L. 
Tabela 6.18: Identificação dos casos influentes para os dados com a perturbação

\begin{tabular}{ccccc}
\hline Observação & Altura & Idade & $K\left(P, P_{(-i)}\right)$ & Calibração \\
\hline 57 & 36.16 & 5.22 & 3.6283 & 0.9998236 \\
\hline
\end{tabular}

Assim, concluímos que a divergência de Kullback-Leibler é um método eficaz para detectar observações influentes. Com a perturbação dos dados, identificamos de fato qual ponto influenciou o ajuste do modelo. 


\section{Capítulo 7}

\section{Conclusões e propostas futuras}

Com os estudos desenvolvidos ao longo deste trabalho, de uma maneira geral, com base nos critérios de seleção de modelos adotados, notamos que o modelo não linear de Brody juntamento com a suposição da distribuição skew normal para a variável resposta foi mais versátil que os demais modelos analisados para ajustar os dados de crescimento de árvores Eucalyptus sp., tanto no caso homocedástico quanto no caso heteroscedástico.

A assimetria dos dados foi modelada utilizando duas distribuições assimétricas, skew normal e skew t. Os modelos com erros seguindo a distribuição skew normal resultaram em um melhor ajuste, quando se comparado aos modelos simétricos.

Quando comparamos os casos homoscedástico e heteroscedástico, para as duas funções de variância utilizada, o modelo heteroscedástico teve melhor resultado. Assim, pode-se notar que a variância dos dados está relacionada a idade das árvores, não descartando a possibilidade da influência de outros fatores que ainda devem ser estudados.

Comprovamos com uma observação contaminada nos dados reais, que a divergência de kullback-Leibler detecta os pontos influentes no modelo. 
Os critérios de seleção de modelos foram validados através de um estudo de simulação feito na seção (6.1).

Como propostas futuras, podemos utilizar outras abordagens, e comparar com a abordagem bayesiana considerada aqui. Outros modelos também podem ser adotados e comparados com os estudados neste trabalho, por exemplo, os modelos não lineares de efeitos mistos, muito úteis quando os dados estão correlacionados.

Considerar outras distribuições para os erros, como a mistura de escala normal (slash, normal contaminada) e fazer o estudo de robustez com a distribuição skew t para os erros.

E por fim, estudar a influência de outros fatores, além da idade, para modelar a heteroscedasticidade, bem como funções mais gerais para o parâmetro de dispersão. 


\section{Referências Bibliográficas}

Arellano-Valle, R. B. (1994). Distribuições Elípticas: Propriedades, Inferência e Aplicações a Modelos de Regressão. Tese de doutorado, Instituto de Matemática e Estatística, Universidade de São Paulo, São Paulo-SP.

Arellano-Valle, R. B. \& Gomez, H. W. (2004). A new class of skew-normal distributions. Communication in Statistics - Theory and Methods, 33, 1465-1480.

Azzalini, A. (1985). A class of distributions which includes the normal ones. Scandinavian Journal of Statistics, 12, 171-178.

Azzalini, A. (2005). The skew-normal distribution and related multivariate families. Scandinavian Journal of Statistics, 32, 159-188.

Azzalini, A. \& Capitanio, A. (1999). Statistical applications of the multivariate skewnormal distribution. Journal of the Royal Statistical Society, 61(3), 579-602.

Azzalini, A. \& Capitanio, A. (2003). Distributions generated by perturbation of symmetry with emphasis on a multivariate skew-t distribution. Journal of the Royal Statistical Society, 65(2), 367-389.

Azzalini, A. \& Dalla Valle, A. (1996). The multivariate skew-normal distribution. Biometrika, 83, 715-726.

Bayes, C. L. \& Branco, M. D. (2007). Bayesian inference for the skewness parameter of 
the scalar skew normal distribution. Brazilian Journal of Probability and Statistics, 21, 141-163.

Bertalanffy, L. V. (1957). Wachstum. in: Helmcke, j.g., h.v.len-gerken und g.starck (ed.): Handbuch der zoologie. Berlin: W. de Gruyter, Bd. 8, 10. Lieferung, pages $1-68$.

Branco, M. D. \& Dey, D. K. (2001). A general class of multivariate skew-elliptical distributions. Journal of Multivariate Analysis, 79, 99-113.

Branco, M. D. \& Dey, D. K. (2002). Regression model under skew elliptical error distribution. The Journal of Mathematical Sciences, 1, 151-169.

Brody, S. (1945). Bioenergetics and growth.

Brooks, S. P. (2002). Discussion on the paper by spiegelhalter, best, carlin and van del linde. Journal Royal of Statistical Society, 64(3), 616-618.

Brown, J. E., Fitzhugh, H. A. \& Cartwright, T. C. (1976). A comparison of nonlinear models for describing weight-age relationships in cattle. Journal of Animal Science, 42(4), 810-818.

Cabral, C. R. B., Bolfarine, H. \& Pereira, J. R. G. (2008). Bayesian density estimation using skew student-t-normal mixtures. Computational Statistics and Data Analysis, $52,5075-5090$.

Cancho, V. G., Lachos, V. H. \& Ortega, E. M. M. (2010). A nonlinear regression model with skew-normal errors. Statistical Papers, 57, 547-558.

Carlin, B. P. \& Louis, T. A. (2001). Bayes and empirical bayes methods for data analysis essays on item response theory. Chapman and Hall, New York.

Chen, M. H., Shao, Q. M. \& Ibrahim, J. (2000). Monte carlo methodos in bayesian computation. Springer-Verlag, New York. 
Cho, H., Ibrahim, J. G., Sinha, D. \& Zhu, H. (2009). Bayesian case influence diagnostics for survival models. Biometrics, 65, 116-124.

Cook, R. D. \& Weisberg, S. (1983). Diagnostics for heteroscedasticity in regression. Biometrika, 70, 1-10.

Cysneiros, F. J. A. \& Vanegas, L. H. (2008). Residuals and their statistical properties in symmetrical nonlinear models. Statistics $\mathscr{6}$ Probability Letters, 78, 3269-3273.

Davidian, M. \& Carrol, R. J. (1987). Variance function estimation. Journal of American Statistical Association, 139, 1079-1091.

De la Cruz, R. \& Branco, M. D. (2009). Bayesian analysis for nonlinear regression model under skewed errors, with application in growth curves. Biometrical journal, 51(4), 588-609.

De Paz, C. C. P., Packer, I. U., De Freitas, A. R., Tambasco-Talhari, D., De Almeida Regitano, L. C., De Alencar, M. M. \& Da Cruz, G. M. (2004). Adjustment of nonlinear models in study of association between genetic polymorphisms and growth in beef cattle. Revista Brasileira de Zootecnia, 33(6), 1416-1425.

Diblasi, A. \& Bowman, A. (1997). Testing for constant variance in a linear model. Statistics 8 Probability Letters, 82, 95-103.

DiCiccio, T. J. \& Monti, A. C. (2004). Inferential aspects of the skew exponential power distribution. Journal of the American Statistical Association, 99, 439-450.

Fekedulegn, D., Mac Siurtain, M. P. \& Colbert, J. J. (1999). Parameter estimation of nonlinear growth models in forestry. Silva Fennica, 33(4), 327-336.

Fernández, C. \& Steel, M. F. J. (1998a). Bayesian regression analysis with scale mixtures of normal. Econometric Theory, 16, 80-101. 
Fernández, C. \& Steel, M. F. J. (1998b). On bayesian modeling of fat tails and skewness. Journal of the American Statistical Association, 93, 359-371.

Ferreira, J. T. A. S. \& Steel, M. F. J. (2006). A constructive representation of univariate skewed distributions. Journal of the American Statistical Association, 101, 604-618.

Ferreira, J. T. A. S. \& Steel, M. F. J. (2007). A new class of skewed multivariate distributions with applications to regression analysis. Statistica Sinica, 17(2), 505529.

Forni, S., Piles, M., Blasco, A., Varona, L., Oliveira, H. N., Lôbo, R. B. \& Albuquerque, L. G. (2007). Analysis of beef cattle longitudinal data applying a nonlinear model. Journal of Animal Science, 85(12), 3189-3197.

Forni, S., Piles, M., Blasco, A., Varona, L., Oliveira, H. N., Lôbo, R. B. \& Albuquerque, L. G. (2009). Comparison of different nonlinear functions to describe nelore cattle growth. Journal of Animal Science, 87(2), 496-506.

Freitas, A. R. (2007). Estimativas de curvas de crescimento na produção animal. Embrapa Pecuária Sudeste, Documento 68, 30p.

Gamito, S. (1998). Growth models and their use in ecological modelling: An application to a fish population. Ecological Modelling, 113(1-3), 83-94.

Gelfand, A. E., Dey, D. K. \& Chang, H. (1992). Model determination using predictive distributions with implementation via sampling-based methods. Bayesian Statistics 4 (Peñiscola,1991).

Genton, M., He, L. \& Liu, X. (2001). Moments of skew-normal random vectors and their quadratic forms. Statistics and Probability Letters, 51, 319-325.

Genton, M. G. (2004). Skew-Elliptical Distributions and Their Applications: A Journey Beyond Normality. Chapman \& Hall/CRC, Boca Raton, FL. 
Gomez, H. W., Venegas, O. \& Bolfarine, H. (2007). Skew-symmetric distributions generated by the distribution function of the normal distribution. Environmetrics, 18, 395-407.

Gompertz, B. (1825). On the nature of the function expressive of the law of human mortality, and a new mode of determining the value of live contengencies. Philosophical Transactions of the Royal Society, 182, 513-585.

Guedes, M. H. P., Muniz, J. A., Perez, J. R. O., Silva, F. F., Aquino, L. H. \& Santos, C. L. (2004). Estudo das curvas de crescimento de cordeiros das raças santa inês e bergamácia considerando heterogeneidade de variâncias. Ciência e Agrotecnologia, 28(2), 381-388.

Gupta, A. K. (2003). Multivariate skew t-distribution. Statistics, 37(4), 359-363.

Gupta, A. K. \& Chen, T. (2001). Goodness of fit tests for the skew-normal distribution. Communication in Statistics, Computation and Simulation, 30, 907-930.

Henze, N. (1986). A probabilistic representation of the skew-normal distribution. Scandinavian Journal of Statistics, 13, 271-275.

Lange, K. L., Little, R. J. \& Taylor, J. M. G. (1989). Robust statistical modelling using the t-distribution. Journal of the American Statistical Association, 84, 881-896.

Lin, J. G., Wei, B. C. \& Zhang, N. S. (2004). Varying dispersion diagnostics for inverse gaussian regression models. Journal of Applied Statistics, 31, 1157-1170.

Lin, J. G., Zhu, L. X. \& Xie, F. C. (2009). Heteroscedasticity diagnostics for t linear regression models. Metrika, $\mathbf{7 0}(1), 59-77$.

Lunn, D. J., Thomas, A., Best, N. \& Spiegelhalter, D. (2000). Winbugs - um quadro de modelagem bayesiana: conceitos, estrutura e capacidade de extensão. Estatística e Computação, 10, 325-337. 
Mazzini, A. R. A., Muniz, J. A., Aquino, L. H. \& Silva, F. F. (2003). Análise da curva de crescimento de machos hereford. Ciência e Agrotecnologia, 27(5), 1105-1112.

Peng, F. \& Dey, D. (1995). Bayesian analysis of outlier problems using divergence measures. The Canadian Journal of Statistics, 23(2), 199-213.

R Development Core Team (2009). R: A Language and Environment for Statistical Computing. R Foundation for Statistical Computing, Vienna, Austria.

Riazoshams, H. \& Midi, H. (2009). A nonlinear regression model for chickens growth data. European Journal of Scientific Research, 35(3), 393-404.

Rocha Sarmento, J. L., Regazzi, A. J., De Sousa, W. H., Torres, R. D. A., Breda, F. C. \& De Oliveira Menezes, G. R. (2006). Analysis of the growth curve of santa ines sheep. Revista Brasileira de Zootecnia, 35(2), 435-442.

Sahu, S. K., Dey, D. K. \& Branco, M. D. (2003). A new class of multivariate skew distributions with applications to bayesian regression models. Canadian Journal of Statistics, 31(2), 129-150.

Santos, V. B. d., Yoshihara, E., Fonseca de Freitas, R. T. \& Neto, R. V. R. (2008). Exponential growth model of nile tilapia (oreochromis niloticus) strains considering heteroscedastic variance. Aquaculture, 274(1), 96-100.

Schwarz, G. E. (1978). Estimating the dimension of a model. Annals of Statistics, 6, $461-464$.

Seber, G. A. F. \& Wild, C. J. (1989). Nonlinear Regression. Probability and Mathematical Statistics. Wiley, New York.

Simonoff, J. S. \& Tsai, C. L. (1994). Improved tests for nonconstant variance in regression based on the modified profile likelihood. Applied Statistics, 43, 357-370. 
Spiegelhalter, D., Thomas, A., Best, N. \& Lunn, D. (2003). WinBUGS user manual, version 1.4. Cambridge: MRC Biostatistics Unit.

Spiegelhalter, D. J., Best, N. G., Carlin, B. P. \& Van Der Linde, A. (2002). Bayesian measures of model complexity and fit (with discussion). Journal Royal of Statistical Society, 64, 583-639.

Tsai, C. L. (1986). Score test for the first-order autoregressive model with heteroscedasticity. Biometrika, 73, 455-460.

Vanegas, L. H. \& Cysneiros, F. J. A. (2010). Assesment of diagnostic procedures in symmetrical nonlinear regression models. Computational Statistics \& Data Analysis, 54, 1002-1016.

Verhulst, P. F. (1938). Notice sur la loi que la population suit dans son accroissement. Corr. Math. Phys, 10, 113-121.

Xie, F. C., Wei, B. C. \& Lin, J. G. (2009). Homogeneity diagnostics for skew-normal nonlinear regression models. Statistics 85 Probability Letters, 79, 821-827.

Zwietering, M. H., Jongenburger, I., Rombouts, F. M. \& Van't Riet, K. (1990). Modeling of the bacterial growth curve. Applied and Environmental Microbiology, 56(6), $1875-1881$. 